\title{
Who is Afraid of Political Instability?
}

\author{
By: Nauro F. Campos and Jeffrey B. Nugent
}

Working Paper Number 326

July 2000 


\title{
WHO IS AFRAID OF POLITICAL INSTABILITY?
}

\author{
by Nauro F. Campos and Jeffrey B. Nugent
}

\section{Executive Summary}

The analysis of the consequences of socio-political instability has been a central theme in recent macroeconomic research, in general, and in the economic growth literature, in particular. There are two different views on the nature of the relationship between political instability and growth. Some authors submit that political instability disrupts productive activities and increases uncertainty. By doing so, it undermines the incentives for the accumulation of physical capital with detrimental consequences for the rate of economic growth. Other economists argue that economic growth leads to more political instability because growth entails substantial structural changes that undo political coalitions and induce painful read*justments in the balance of power among different interest groups.

Despite the negative relationship between political instability and economic growth having been elevated to "stylized fact" status, the empirical studies on which this assessment is based have been heavily criticized for ad hoc selection of explanatory variables, excessively narrow definitions of political instability, insufficient sensitivity analysis and failure to investigate the direction of causality. Although not fully sharing this criticism, we believe that this finding should not be elevated to "stylized fact" status without demonstrating that causality exists and runs from political instability to growth, rather than vice-versa.

The objective of this paper is to investigate the existence and direction of a causal relationship between political instability and economic growth. To do so, we construct two indexes of political instability (one for mild and the another for severe instability) for non-overlapping five-year periods, between 1960 and 1995, for 98 developing countries. We use the Granger causality framework and report Anderson-Hsiao-Arellano instrumental variable estimates.

We find no evidence of the hypothesized negative and causal relationship between political instability and economic growth. Our sensitivity analysis, however, suggests two possible explanations for the apparent disagreement between our findings and those of the rest of the literature. First, for the full sample, the negative relationship obtains only contemporaneously (and independently of whether we use 25- or 5-year averages). Second, in the long run and ignoring institutional factors, the Sub-Saharan Africa sample seems to be the driving force in arriving at the negative relation between growth and political instability. 


\title{
WHO IS AFRAID OF POLITICAL INSTABILITY?*
}

\author{
Nauro F. Campos \\ CERGE-EI, Charles University, \\ Centre for Economic Policy Research \\ (CEPR), and William Davidson Institute at \\ the University of Michigan
}

\author{
Jeffrey B. Nugent \\ Department of Economics \\ University of Southern California
}

This version: July 15, 2000

\begin{abstract}
An unstable macroeconomic environment is often regarded as detrimental to economic growth. Among the sources contributing to such instability, the literature has assigned most of the blame to political issues. This paper empirically tests for a causal and negative long-term relation between political instability and economic growth, but finds no evidence of such a relationship. Sensitivity analysis indicates that there is a contemporaneous negative relationship and that, in the long run and ignoring institutional factors, the Sub-Saharan Africa group plays the determining role in steering this relationship into causal and negative.
\end{abstract}

Keywords : economic growth, political instability. JEL classification: O40, E23, D72.

\begin{abstract}
* We thank Daron Acemoglu, Lee Alston, Sebastian Edwards, Yi Feng, Dipak Gupta, Jan Hanousek, Phil Hoffman, Stephen Haber, Steven Helfand, Cheng Hsiao, Phil Keefer, Jan Kmenta, Christian Morrisson, Bernardo Mueller, Lant Pritchett, James Robinson, Gerard Roland, Luis Serven, Jakob Svensson, Kenneth Sokoloff, Viatcheslav Vinogradov, Ronald Wintrobe, two anonymous referees and seminar participants at Stanford University, University of Calabria and Stockholm School of Economics, WEA (Seattle), LACEA (Bogota) and EPCS (Gothenburg) meetings for valuable comments on previous versions of this paper. Alacritous research assistance was provided by Aurelijus Dabusinskas. The usual disclaimer applies. The data set used in this paper is available from the authors upon request, and it is also available on-line as http://home.cerge.cuni.cz/ncampost/gispi.txt.
\end{abstract}

Nauro F. Campos, CERGE-EI, P.O. Box 882, 11121 Prague 1, Czech Republic. E-mail: nauro.campos@cerge.cuni.cz.

Jeffrey B. Nugent, Department of Economics, University of Southern California, Los Angeles, CA 90089-0253, USA. E-mail: nugent@ rcf.usc.edu 


\section{Introduction}

The analysis of the consequences of socio-political instability (hereafter, SPI) has been a central theme in recent macroeconomic research, in general, and in the economic growth literature, in particular. ${ }^{1}$ There are two very different views on the relationship between SPI and growth. Some submit that SPI disrupts production and increases uncertainty in the economy. By doing so, it undermines the incentives for the accumulation of physical capital and reduces the rate of economic growth. ${ }^{2}$ Others argue that economic growth leads to either higher SPI (because growth entails substantial structural changes that undo political coalitions and induce painful readjustments in the balance of power among different interest groups ${ }^{3}$ ) or lower SPI because it reduces social and political tensions. Despite the negative relationship between SPI and economic growth having been elevated to "stylized fact" status, ${ }^{4}$ the empirical studies on which this is based have been heavily criticized for ad hoc selection of explanatory or control variables, excessively narrow definitions of SPI, insufficient sensitivity analysis and failure to investigate the direction of causality. ${ }^{5}$ Although not fully sharing this criticism, we

1 Among the variables allegedly affected by SPI are the independence of central banks (see e.g. Cukierman, Webb and Neypati, 1992 and Cukierman and Webb, 1995), seigniorage (Cukierman, Edwards, and Tabellini, 1992), aggregate investment (Ozler and Rodrik, 1992), budget deficits (Roubini, 1991), external debt (Alesina and Tabellini, 1989, and Ozler and Tabellini, 1991), and exchange rate regime (Collins, 1996). Examples of the literature on SPI and growth are Barro (1991), Alesina, Ozler, Roubini and Swagel (1996), and Ades and Chua (1997).

${ }^{2}$ See Alesina, Ozler, Roubini and Swagel (1996), and references therein.

${ }^{3}$ See, e.g., Olson (1963) and North (1981).

4 According to the review of the literature by Mankiw (1995, 302), a robust finding is that "political instability, as measured by the frequency of revolutions, coups, or wars, is negatively associated with growth". Similarly, Persson and Tabellini (1999) conclude in their chapter for the Handbook of Macroeconomics (1999): "Political instability, as measured by more frequent regime changes, or politic al unrest and violence, is significantly and negatively correlated with growth in cross-country data".

5 Durlauf and Quah (1998) provide the most extensive review of this empirical literature and find that more than 80 different explanatory variables have been used thus far. 
believe that this finding should not be elevated to "stylized fact" status without demonstrating that causality exists and runs from SPI to growth, rather than vice-versa.

The objective of this paper is to investigate the existence and direction of a causal relationship between SPI and economic growth. To do so, we construct two different indexes of SPI for nonoverlapping five-year periods, between 1960 and 1995, for 98 developing countries. We use the Granger causality framework and report Anderson-Hsiao-Arellano instrumental variable estimates.

We find no evidence of the hypothesized negative and causal relationship between political instability and economic growth. Our sensitivity analysis, however, suggests two explanations for the apparent disagreement between our findings and those of the rest of the literature. First, for the full sample, the negative relationship obtains only contemporaneously (and independently of whether we use 25- or 5-year averages). Second, in the long run and ignoring institutional factors, the Sub-Saharan Africa sample seems to be the driving force in arriving at the negative relation between SPI and growth.

The paper is organized as follows. In Section II we present our two measures of SPI, describe how each index is constructed and map the relationship between them. In Section III we discuss the advantages and shortcomings of the Granger-causality framework. In Section IV we present our causality results, reporting Anderson-Hsiao-Arellano instrumental variable estimates. In Section V we subject these results to sensitivity analysis. Section 6 concludes.

\section{The Measurement of Political Instability}

There seem to be wo rather different understandings of SPI in the literature. One stresses regular and irregular government transfers, the other much harsher aspects, such as revolutions, coups 
d'Etat, civil wars and political assassinations. $^{6}$ That these overlap (e.g., by including irregular government transfers) does little to diminish the different intensities that each attaches to "instability." While the former interpretation constrains it to relatively tame phenomena, the latter places it closer to social chaos. In order to recognize both views, we construct two measures of SPI, one capturing the more severe and the other the less severe forms of SPI. While many other variants could have been used, our justification is that these can be considered the bounds of the realistic range of such measures, together permitting a more complete depiction of the causality structure between SPI and growth.

Our measure of "severe" or "upper-bound" SPI follows existing literature in using three indicators: the numbers of political assassinations per million people, revolutions and successful coups d'Etat. $^{7}$ The first of these is especially important because it captures a magnitude dimension that is largely missing from the other (frequency) measures.

For the measure of the "moderate" or "lower-bound" SPI we follow Chen and Feng (1996) and others in the use of indicators from the Polity III data collection (Jaegger and Gurr, 1996). A crucial advantage of using this source is its relatively complete country and time coverage. From it, we select the following variables: competitiveness and regulation of political participation; regulation, competitiveness, and openness of executive recruitment; and the legal (de jure) and operational (de facto) independence of the chief executive. ${ }^{8}$ Because political actors and processes are to be subject to systematic regulation, this set of indicators is capable of capturing the extent of even subtle changes in

\footnotetext{
${ }^{6}$ See footnote 4 above.

${ }^{7}$ The data source is Barro and Lee (1993).

${ }^{8}$ Although a more appropriate lower-bound measure of SPI might include strikes, demonstrations without violence or deaths, regional and internal conflicts, free press, etc., to our knowledge, data on such variables is largely lacking for our sample (98 developing countries, 1960-1995).
} 
both legal and actual practice. The less regulated are such actors and processes, the greater is the potential for social and political change (and the higher the value of this SPI index). ${ }^{9}$

These two SPI indexes are constructed by the method of principal components. This method has the benefit that it addresses the latent variable problem and minimizes the inherent arbitrariness in the aggregation procedure. For the severe or upper bound SPI (UBSPI) indicator, the loadings resulting from this procedure are 0.3162 for assassinations, 0.6909 for revolutions, and 0.6502 for coups. In the case of lower bound SPI (LBSPI), the resulting loadings are 0.3923 and 0.1105 for the competitiveness and regulation of political participation (respectively); $0.4677,0.4734$ and 0.3535 for regulation, competitiveness, and openness of executive recruitment; and 0.2317 and 0.4608 for the legal (de jure) and operational (de facto) independence of the chief executive.

Since both indexes are measures of SPI but capturing quite different aspects of it, one would expect them to be positively but not highly correlated. In general, this expectation is fulfilled: with the exception of the Middle East and North Africa region, for all other regions the correlation between the respective pairs of SPI indexes is positive and statistically significant but less than 0.6 (as shown in column 1, Table 1). Since these correlation coefficients are only with respect to the linear relation between the two measures, to get at non-linearities, in the remaining columns of Table 1 we present some results from two alternative specifications. Column (2) contains the adjusted- $\mathrm{R}^{2}$ of regressions where the dependent variable is LBSPI and the independent variables are UBSPI and its square and column (3) those where a cubic term is added. Note that the adjusted values of $R^{2}$ are universally higher when the cubic terms are included. In the remaining columns of the table are the regression coefficients

\footnotetext{
${ }^{9}$ Since in Polity III (Jaegger and Gurr, 1996) countries receive high scores when the extent of regulations is high (implying low SPI), for present purposes the coding has been reversed.
} 
estimated from the model of column (3). Note that for the sample as a whole and for each region, the coefficients of the linear, quadratic and cubic terms have alternating signs - indicating non-linearity in the relationship - and are, with a single exception, significant at the 1-percent level. On this basis, we claim that our lower-bound SPI index is indeed systematically, though non-linearly, related to SPI of the more traditional "severe" or upper-bound variety.

\section{INSERT TABLE 1 ABOUT HERE}

Next we turn to the rate of real per capita GDP growth and the other variables used in the analysis to follow and to the time periods chosen. All such measures are collected for non-overlapping five-year periods, covering the period 1960-1995 in an unbalanced panel of 98 developing countries. ${ }^{10}$ There are 14 countries from Asia, 21 from Latin America, 17 from the Middle East and North Africa and 46 from Sub-Sahara Africa. Table 2 shows basic statistics and correlation matrix. The negative correlation coefficients between both UBSPI and LBSPI and growth are both statistically significant for the full sample and for each of the regions. We also found that the same significant negative relationship holds for the pure cross section relating to growth over the whole period as to that reported in the table with the pooled data for five-year intervals. However, this negative relationship need not imply causality, the issue explored below.

\footnotetext{
${ }^{10}$ Per capita GDP data are from Summers and Heston (1994). We chose an unbalanced panel in order to deviate as little as possible from the rest of the literature. The differences in country and time coverage between our sample and those used in other studies are marginal.
} 


\section{INSERT TABLE 2 ABOUT HERE}

\section{The Costs and Benefits of Granger Causality}

This section discusses the conceptual and econometric advantages (as well as the limitations) of the Granger-causality framework. This framework has endured the test of time because of its elegance and strong intuitive appeal: the notion that an event in the future cannot cause one in the past. ${ }^{11}$ Consider two time series, $x_{t}$ and $y_{t}$. Series $x_{t}$ is said to Granger-cause series $y_{t}$ if, in a regression of $y_{t}$ on lagged $y$ 's and lagged $x$ 's, the coefficients of the lagged $x$ 's are jointly significantly different from zero.

There are two critical issues to be addressed in conducting Granger causality tests. ${ }^{12}$ The first concerns the length and frequency of the time lags. On their length, Granger warns that "using data measured over intervals much wider than actual causal lags can destroy causal interpretation" (Granger, 1987, p.49). We believe that five year periods are short enough to allow us to investigate the effects of lagged variables, and yet long enough to be meaningful for studying the long-run effects of SPI on economic growth, and vice-versa (Solow, 1997). As to their frequency, there are tests to determine the optimal number of lags. Yet, because ours is a short panel, we use the grid procedure identified below to evaluate the robustness of the results presented below.

The second issue to be dealt with lies in the information set. The Granger test depends on the assumption that the cause contains unique information about the effect, in the sense that it is exhaustive

\footnotetext{
${ }^{11}$ Granger remarks that "causation is a non-symmetric relationship, and there are various ways in which asymmetry can be introduced, the most important of which are controllability, a relevant theory, outside knowledge, and temporal priority" (1987, 49.) For discussion see, e.g., Hsiao (1979), and Zellner (1989).

${ }^{12}$ We do not know of other studies that use the Granger framework in this context. The closest paper to ours is Blomstrom, Lipsey and Zejan (1996).
} 
and unavailable elsewhere. If the two variables in the Granger test are affected by a third one, unless the latter is accounted for, the test can be rendered useless. In what follows, we present Granger causality results that are unaffected by the inclusion of variables that could potentially play this disruptive role.

Finally, we must attend to the econometric issue that arises from the inclusion on the right-hand side of the (lagged) dependent variable, referred to in the econometric literature as the dynamic panel problem: unless the time dimension of the panel is very large, parameter estimates will be inconsistent and biased. ${ }^{13}$ While the best solution to this problem is still an object of debate in the econometrics literature, ${ }^{14}$ in one of the few studies focusing on "short and wide" panels (like ours), Kiviet (1995) finds that the instrumental variable approach pioneered by Anderson and Hsiao (1982) performs as well as any other alternative. Hence, we use this method. Specifically, we first-differenced all variables and followed Arellano's (1989) recommendation by using the twice-lagged levels instead of the twicelagged first-differences as instruments.

\section{Empirical Results}

We begin our investigation of the causality patterns between SPI and economic growth in Tables 3 and 4. In Table 3 we ask whether (severe or moderate) SPI Granger-causes per capita GDP growth. In our complete sample of 98 developing countries, we find no evidence of a causal relationship: neither moderate nor severe SPI seems to Granger-cause economic growth. When we break down these results by region, at best we find a negative relationship between moderate SPI and

\footnotetext{
${ }^{13}$ For discussion see, e.g., Hsiao (1986), Sevestre and Trognon (1992), and Baltagi (1995).

${ }^{14}$ See, among others, Holtz-Eakin et al. (1988), Arellano and Bond (1991), Kiviet (1995), and Judson and Owen (1999).
} 
growth that is significant at the .10 (but not .05) level and only for the Sub-Saharan Africa sample. Note also that the effect (i.e., the sign of the relevant coefficient) varies substantially not only by region, but also by the SPI index used. In particular, for the Middle East and North Africa region, the commonly used severe SPI index Granger-causes greater rather than lower rates of economic growth. ${ }^{15}$ With respect to the relationship flowing from economic growth to SPI, the results presented in Table 4 fail to reveal any indication of causality.

\section{INSERT TABLES 3 AND 4 ABOUT HERE}

Summarizing, the evidence supporting the hypothesis that SPI causes a decrease in the growth rate of per capita income seems much weaker than generally believed. In addition, such a negative and causal relation seems to be largely confined to the Sub-Saharan Africa sample, the only sample for which the relevant coefficient is statistically significant. Finally, we find no evidence whatsoever of causality flowing the other way (i.e., from per capita GDP growth to SPI). Before discussing these results further, in the following section we subject them to various sensitivity analyses.

\section{Sensitivity Analysis}

The objective of this section is to test the sensitivity of the results to various modifications, which (in the interest of space) are only partially reported in the text.

${ }^{15}$ Campos, Nugent and Robinson (1999) find that in the Middle East and North Africa region, external political instability affect economic performance directly and indirectly (via policy distortions), conditional on the level of internal SPI. Controlling for external political instability renders the coefficient on internal political instability (as above) statistically insignificant. 
The first sensitivity test reported here is with respect to the frequency of the time lags. Having in mind that ours is a short panel, we experimented with including two lags of the "causing variable $x$ " (instead of the one lag results presented throughout this paper), and with the exclusion of one lag of the "caused variable $y$." Since none of these changes affects our conclusions, and our focus is on the relation between SPI and long-term growth, in subsequent sensitivity analyses we keep the length of the lag fixed at five years. ${ }^{16}$

The issue regarding the content of the information set refers to whether there are omitted variables that affect both growth and SPI. A particularly promising candidate for such a role is institutional development. Our measure of institutional development is the index of "legislative effectiveness" from Banks (1984). It is selected here because it is available for a large number of developing countries for a long period of time, and conceptually it captures an aspect of institutional development that is closely related to SPI. ${ }^{17}$ The unattractive features are that the data are available only until 1984 (thereby forcing us to lose observations) and it is a categorical variable that assumes one of four values, from zero to $3 .{ }^{18}$ We mitigate these drawbacks by lagging it one period and using 5-year

\footnotetext{
${ }^{16}$ It would be important to investigate the effect of alternative lag lengths (such as one, two, three or four year periods) relative to the five year length use here. While Gupta (1990) constructed annual series for a similar SPI index, he did so only until 1982 and in personal communication has stated that the updating of these series (until 1995) has not yet been completed. We thus have to leave this important exercise for future work.

${ }^{17}$ For example, the quality of the bureaucracy is another aspect of institutional development, but its relation to SPI is not as direct or clear.

18 "Legislative effectiveness" (LEGEF) is coded zero if no legislature exists, ' 1 ' if legislative activity is of a "rubber stamp" character, its implementation is faulty or it is completely subordinate to the executive, ' 2 ' if the executive's power substantially outweighs, but does not completely dominate, that of the legislature, and ' 3 ' for an "effective legislature" distinguished by significant governmental autonomy, including its ability to override vetoes by the executive.
} 
averages. Our working hypothesis is that, in a given country, the level of SPI is contemporaneously negatively correlated with the level of institutional development. ${ }^{19}$

The initial evel of per capita income is another natural candidate for having an influence on both SPI and economic growth. The convergence property of the neoclassical growth model suggests that growth should be negatively related to the initial level of income per capita. We conjecture also that lower levels of per capita income may increase the potential for political instability.

In what follows, we present results obtained by adding the levels of both initial income and institutional development to the specifications for the Granger-causality tests reported in the previous section. In Table 5 we investigate whether or not SPI Granger-causes GDP growth, once we control for the levels of institutional development and initial income. There are two important differences in results from those in Table 3. First, a rise in lower bound SPI ceases to Granger-cause (a decrease in) economic growth in Sub-Saharan Africa and, second, a rise in severe SPI ceases to Granger-cause (an increase in) GDP growth in the Middle East and North Africa. It is worth noting that it is the inclusion of the institutional variable that makes the coefficient for Sub-Saharan Africa statistically insignificant but it is the inclusion of initial income per capita that does so for the Middle East and North Africa. In other words, the result for Sub-Saharan Africa from Table 3 holds with initial income in the specification (provided the institutional development variable is not included) and the result for the MENA region holds with the institutional development in the specification (provided initial income is not included).

${ }^{19}$ We find support for the hypothesis that high levels of SPI are associated with low levels of institutional development. The contemporaneous correlation between "legislative effectiveness" and each of our SPI indexes are negative and statistically significant, at the 5 percent level, for our whole sample and each of the four regions individually. 


\section{INSERT TABLES 5 AND 6 ABOUT HERE}

In Table 6 we ask whether economic growth Granger-causes SPI after controlling for the levels of institutional development and initial income. Recall that from Table 4 there was no indication of causality flowing in this direction, irrespective of the SPI index used or of the regional breakdown. These same results hold in Table 6 with one exception. The coefficient on economic growth for Latin America turns out to be statistically significant after we enlarge the information set. Moreover, the result indicates that a rise in the rate of per capita economic growth in this region seems to Granger-cause a rise in the level of our moderate index of SPI. Further investigation revealed that it is the inclusion of the institutional development variable that is responsible for this change. ${ }^{20}$ The identification of the precise mechanism for this destabilizing effect of economic growth in Latin America is left for future work.

Further sensitivity tests were performed for alternative control variables with the following results (available on request from the authors). In particular, the results are not affected by using (instead of level of per capita income or institutional development) the rate of population growth or the growth rate of the country's main trade partners as alternative controls. The use of a "terms of trade" index as an alternative control does have some effect, but this is to make the coefficient on LBSPI for the Africa region statistically insignificant, thereby further strengthening the case against a causal relation.

Finally, some tests with respect to estimation procedure were also performed. Yet, our conclusions remain the same even if instead of the Anderson-Hsiao-Arellano estimator, we should use

\footnotetext{
${ }^{20}$ Notice that including only initial income in the relevant specifications also makes the coefficient on economic growth become statistically significant, although only marginally at the 10 percent level. It is on this basis that we claim that the institutional variable is responsible for the change.
} 
any of the following procedures: OLS (with levels), OLS (with first-differences), the Anderson-Hsiao estimator, the one-step GMM estimator proposed by Arellano and Bond (1991), the two-step GMM estimator proposed by Arellano and Bond (1991), or the GMM estimator proposed by Ahn and Schmidt (1995).

\section{Conclusions}

The objective of this paper was to investigate the existence (and direction) of a causal relationship between SPI and economic growth. We find that the evidence supporting the hypothesis that high levels of SPI cause lower rates of economic growth is much weaker than generally believed, as we find no traces of a long-run causal relationship. How can this be reconciled with the results from other studies? Our sensitivity analysis shows that the Sub-Sahara Africa sample constitutes a large part of the explanation. Not only is the Sub-Saharan African sample much larger than those for other regions, but also its SPI seems to be of a more structural nature. This explanation is supported by our finding that, once one controls for institutional development or alternatively the terms of trade, the causality results vanish. Hence, we suspect that of other studies were to exclude African countries from their samples, the existing results of a negative relation between SPI and growth would disappear.

Given the prominence attached to SPI in recent macroeconomic research in general (and in political economy in particular), there are a number of suggestions for further research that should be put forward. First, in light of the inconsistency between existing results (of a negative contemporaneous relation between SPI and economic growth) and our own findings of the lack of a causal negative relationship between SPI and growth, one should ask at what frequencies and lag lengths does the 
relationship change from non-causal to causal? ${ }^{21}$ As noted above, this is an important question answerable only when higher frequency data should become available.

A second direction for future research would be to investigate whether a causal negative relationship emerges between growth and other important sources of instability, for instance, policy variability. Hopenhayn and Muniagurria (1996) have formalized its macroeconomic role, and Brunetti (1998) presents relevant empirical evidence.

Third, there should be considerable scope to identify additional omitted variables, especially those of an institutional nature, which might be related to both SPI and growth. Numerous institutional variables may be relevant, like the fairness and effectiveness of the judicial system and the stability of property rights. Indeed, in a cross-sectional framework Keefer and Knack (1995) find that, once these are taken into account, the megative effect of SPI on growth vanishes. Another important candidate for such an omitted variable role, following Persson and Tabellini $(1992,1994)$ and Alesina and Perotti (1996), might be the level of income inequality. Unfortunately, the data (on income distribution and institutions) needed for these "enlargements" of our Granger tests are not presently available.

Fourth, given the difficulties in constructing a lower-bound measure of socio-political instability, exploratory research of this sort with other SPI measures should be encouraged.

Finally, in the light of the wide variety of other consequences that have been alleged to SPI, and referred to in our introduction, serious consideration should also be given to the examination of causal

${ }^{21}$ For example, might it not be that there is a very short period of an initial negative impact of SPI on growth, say six months or a year, followed by several years of catch-up, reflected in a positive relation between five year lagged SPI and growth? 
relationships between SPI and these other variables. In particular, it would be interesting to see whether the Sub-Saharan Africa sample would again play such a determinant role. 


\section{References}

Ades, A. and H. Chua (1997). "Thy Neighbor's Curse: Regional Instability and Economic Growth," Journal of Economic Growth 2, 279-304.

Alesina, A., S. Ozler, N. Roubini and P. Swagel. (1996). "Political Instability and Economic Growth," Journal of Economic Growth 1, 189-211.

Alesina, A. and R. Perotti. (1996). "Income Distribution, Political Instability, and Investment," European Economic Review 40, 1203-1228.

Alesina, A, and G. Tabellini. (1989). "External Debt, Capital Flight and Political Risk," Journal of International Economics 27, 199-220.

Anderson, T.W. and C. Hsiao (1982). "Formulation and Estimation of Dynamic Models using Panel Data," Journal of Econometrics 18, 47-82.

Arellano, M. (1989). "A Note on the Anderson-Hsiao Estimator for Panel Data," Economic Letters 31, 337-341.

Arellano, M. and S. Bond (1991). Some Tests of Specification for Panel Data: Monte Carlo Evidence and an Application to Employment Equations, Review of Economic Studies 58, 277-297.

Baltagi, B. (1995). Econometric Analysis of Panel Data. New York: John Wiley \& Sons.

Banks, A. (1984). Political Handbook of the World. New York: McGraw-Hill.

Barro, R. (1991), "Economic Growth in a Cross Section of Countries," Quarterly Journal of Economics 106, 407-444.

Barro, R. and J. Lee. (1993)."Data set for 138 Countries," (available by anonymous ftp from www.nber.org, at directory pub/barro.lee).

Blomstrom, M., Lipsey, R. and M. Zejan. (1996).“Is Fixed Investment the Key to Economic Growth?” Quarterly Journal of Economics 111, 269-276.

Brunetti, A. (1998), "Policy Volatility and Economic Growth: A Comparative, Empirical Analysis", European Journal of Political Economy 14, 35-52.

Campos, N., Nugent, J. and J. Robinson. (1999). "Can Political Instability Be Good for Growth? The Middle East and North Africa Case" in I. Limam (ed.), Institutional Reform and Development in the MENA Region, Cairo: Economic Research Forum. 
Chen, B. and Y. Feng. (1996). "Some Political Determinants of Economic Growth: Theory and Empirical Implications," European Journal of Political Economy 12, 609-627.

Collins, S.(1996). “On Becoming More Flexible: Exchange Rate Regimes in Latin America and the Caribbean," Journal of Development Economics 51,117-138.

Cukierman, A., Edwards, S. and G. Tabellini. (1992). "Seigniorage and Political Instability," American Economic Review 82, 537-555.

Cukierman, A. and S. Webb (1995). "Political Influence on the Central Bank", World Bank Economic Review 9, September,

Cukierman, A., Webb, S. and B. Neyapti. (1992). "Measuring the Independence of Central Bank and its Effects on Policy Outcomes,” World Bank Economic Review 6, 353-598.

Durlauf, S. and D. Quah (1998). The New Empirics of Economic Growth, Discussion Paper 384, London: Centre for Economic Performance.

Granger, C.W. (1987). "Causal Inference," The New Palgrave: Econometrics, New York: W.W. Norton.

Gupta, D. (1990). The Economics of Political Violence, New York: Praeger.

Holtz-Eakin, D., Newey, W. and H. Rosen (1988). Estimating Vector Autoregressions with Panel Data, Econometrica 56, 1371-1395.

Hopenhayn, H. and M. Muniagurria (1996), "Policy Variability and Economic Growth," Review of Economic Studies 63, 611-625.

Hsiao, C. (1979). “Causality Tests in Econometrics," Journal of Economic Dynamics and Control 1, 321-346.

Hsiao, C. (1986). Analysis of Panel Data. Cambridge: Cambridge University Press.

Jaegger, K. and T. Gurr. (1996). "Polity III" (available by anonymous ftp from isere.colorado.edu, at directory pub/datasets/polity3).

Judson, R. and A. Owen (1999). Estimating Dynamic Panel Data Models: A Guide for Macroeconomists, Economic Letters 65, 9-15.

Keefer, P. and S. Knack (1995). Institutions and Economic Performance: Cross-Country Tests Using Alternative Institutional Measures, Economics and Politics 7, 207-227. 
Kiviet, J. (1995). On Bias, Inconsistency, and Efficiency of Various Estimators in Dynamic Panel Data Models, Journal of Econometrics 68, 53-78.

Mankiw, G. (1995). “The Growth of Nations," Brookings Papers on Economic Activity 1, 275-310.

North, D. (1981). Structure and Change in Economic History. New York: W.W.Norton.

Olson, M. (1963). "Rapid Growth as a Destabilizing Force," Journal of Economic History 23, 529552.

Ozler, S. and G. Tabellini. (1991). "External Debt and Political Instability," NBER Working Paper 3772 .

Ozler, S. and D. Rodrik (1992). "External Shocks, Politics, and Private Investment: Some Theory and Empirical Evidence," Journal of Development Economics 39, 141-162.

Persson, T. and G. Tabellini (1992), Growth, Distribution, and Politics, in A.Cukierman, Z.Hercowitz and L.Leiderman, eds, Political economy, growth, and business cycles. Cambridge: MIT Press.

Persson, T. and G. Tabellini (1994), Is Inequality Harmful for Growth?, American Economic Review 84, 600-621.

Persson, T. and G. Tabellini (1999). "Political Economics and Macroeconomic Policy", in J. Taylor and M. Woodford, eds. Handbook of Macroeconomics, North-Holland, Amsterdam.

Roubini, N. (1991) "Economic and Political Determinants of Budget Deficits in Developing Countries," Journal of International Money and Finance 10 (Supplement), S49-S72.

Sevestre, P. and A. Trognon (1992). "Linear Dynamic Models" in L. Matyas and P.Sevestre (eds) The Econometrics of Panel Data, Dordrecht: Kluwer.

Solow, R. (1997). Learning from “Learning by Doing”: Lessons for Economic Growth, Stanford: Stanford University Press.

Summers, R. and A. Heston (1994). "Penn World Tables, Version 5.6," (available by anonymous ftp from www.nber.org, at pub/pwt56).

Zellner, A. (1989). “Causality and Causal Laws in Economics,” Journal of Econometrics 39, 7-22. 


\section{Table 1.}

The Relationship between the Lower-Bound and Upper-Bound Indexes of SPI

\begin{tabular}{|c|c|c|c|c|c|c|}
\hline & $\begin{array}{c}\text { Simple } \\
\text { Correlation }\end{array}$ & Adj. $R^{2}$ & Adj. $R^{2}$ & UBSPI & $\mathrm{UBSPI}^{2}$ & $\mathrm{UBSPI}^{3}$ \\
\hline & (1) & (2) & (3) & (4) & (5) & (6) \\
\hline All LDCs & $\begin{array}{c}0.319 \\
{[0.0001]}\end{array}$ & 0.2577 & 0.5616 & $\begin{array}{c}-1.93816^{* * *} \\
(-10.043)\end{array}$ & $\begin{array}{c}2.89843 * * * \\
(22.225)\end{array}$ & $\begin{array}{c}-0.40813 * * * \\
(-16.989)\end{array}$ \\
\hline Asia & $\begin{array}{c}0.284 \\
{[0.0001]}\end{array}$ & 0.3840 & 0.6776 & $\begin{array}{l}-0.73461 \\
(-1.632)\end{array}$ & $\begin{array}{c}4.03200 * * * \\
(10.714)\end{array}$ & $\begin{array}{c}-0.94505^{*} * * \\
(-7.459)\end{array}$ \\
\hline Latin America & $\begin{array}{c}0.535 \\
{[0.0001]} \\
\end{array}$ & 0.3198 & 0.6555 & $\begin{array}{c}-1.08082 * * * \\
(-3.085)\end{array}$ & $\begin{array}{c}3.61378 * * * \\
(13.113)\end{array}$ & $\begin{array}{c}-0.65841 * * * \\
(-10.019)\end{array}$ \\
\hline $\begin{array}{l}\text { Middle East \& } \\
\text { North Africa }\end{array}$ & $\begin{array}{c}-0.027 \\
{[0.8169]}\end{array}$ & 0.1590 & 0.5978 & $\begin{array}{c}-2.91247 * * * \\
(-6.236) \\
\end{array}$ & $\begin{array}{c}3.44408 * * * \\
(10.163)\end{array}$ & $\begin{array}{c}-0.42997 * * * \\
(-8.859) \\
\end{array}$ \\
\hline $\begin{array}{l}\text { Sub-Saharan } \\
\text { Africa }\end{array}$ & $\begin{array}{c}0.319 \\
{[0.0001]}\end{array}$ & 0.3419 & 0.6119 & $\begin{array}{c}-1.45044 * * * \\
(-4.925)\end{array}$ & $\begin{array}{c}3.74465 * * * \\
(15.281)\end{array}$ & $\begin{array}{c}-0.72011 * * * \\
(-11.110)\end{array}$ \\
\hline
\end{tabular}

Notes: Column (1) contains the simple correlation coefficients between the two indexes of sociopolitical instability (LBSPI and UBSPI). Numbers in brackets are p-values.

Column (2) shows the adjusted $\mathrm{R}^{2}$ of a regression where the dependent variable is LBSPI and the independent variables are UBSPI and $\mathrm{UBSPI}^{2}$. In all regressions, both coefficients are significant at the 1 percent level, and have negative and positive signs respectively.

Column (3) shows the adjusted $R^{2}$ of a regression where the dependent variable is LBSPI and the independent variables are UBSPI, $\mathrm{UBSPI}^{2}$ and $\mathrm{UBSPI}^{3}$.

Columns (4), (5) and (6) contain the coefficients on UBSPI, UBSPI ${ }^{2}$ and $\mathrm{UBSPI}^{3}$ for the regression which adjusted $\mathrm{R}^{2}$ is shown in column (3). Numbers in parenthesis are t-statistics. A * denotes that the coefficient is statistically significant at the 10 percent level, $* *$ that it is statistically significant at the 5 percent level, and $* * *$ that it is statistically significant at the 1 percent level. 
Table 2.

Basic Statistics and Correlation Matrix

\begin{tabular}{||l|c|c|c|c|}
\hline \multicolumn{1}{|c|}{ Variable } & Mean & Std.Dev. & Min & Max \\
\hline Growth & 1.5713 & 4.1372 & -15.8808 & 10.6604 \\
\hline UBSPI & -.0472 & 1.2155 & -.9076 & 4.8948 \\
\hline LBSPI & 5.1137 & 1.5630 & .4046 & 10.3362 \\
\hline $\begin{array}{l}\text { Institutional } \\
\text { Development }\end{array}$ & 1.2979 & .9106 & 0 & 3 \\
\hline Per capita income & 2013.7 & 1563.4 & 322 & 7777 \\
\hline Population growth & 2.532 & .7744 & .1490 & 6.9542 \\
\hline $\begin{array}{l}\text { Trading partners' } \\
\text { Growth }\end{array}$ & 2.5198 & 1.2869 & -2.2389 & 6.4387 \\
\hline Terms of Trade & .00088 & .02485 & -.0805 & .1493 \\
\hline
\end{tabular}

Correlation Matrix

\begin{tabular}{||l|c|c|c|c|l|l|l||}
\hline \multicolumn{1}{|c|}{ Variable } & Growth & UBSPI & LBSPI & $\begin{array}{l}\text { Institutional } \\
\text { Dev. }\end{array}$ & $\begin{array}{l}\text { Per } \\
\text { capita } \\
\text { income }\end{array}$ & $\begin{array}{l}\text { Pop. } \\
\text { growth }\end{array}$ & $\begin{array}{l}\text { Trading } \\
\text { partners } \\
\text { Growth }\end{array}$ \\
\hline UBSPI & -0.1168 & & & & & & \\
\hline LBSPI & -0.0610 & 0.3658 & & & & & \\
\hline $\begin{array}{l}\text { Institutional } \\
\text { Development }\end{array}$ & 0.0027 & -0.3899 & -0.6902 & & & & \\
\hline $\begin{array}{l}\text { Per capita } \\
\text { income }\end{array}$ & -0.0954 & -0.1080 & -0.2435 & 0.2245 & & & \\
\hline $\begin{array}{l}\text { Population } \\
\text { growth }\end{array}$ & -0.0992 & -0.0665 & 0.1705 & -0.1404 & -0.0511 & & \\
\hline $\begin{array}{l}\text { Trading } \\
\text { partners' } \\
\text { Growth }\end{array}$ & 0.4006 & -0.0149 & -0.0902 & 0.0176 & -0.1583 & 0.0297 & \\
\hline $\begin{array}{l}\text { Terms of } \\
\text { Trade }\end{array}$ & 0.0980 & 0.0252 & 0.0265 & -0.0645 & 0.0333 & 0.0795 & 0.0596 \\
\hline Note: See text for details. & \multicolumn{7}{|l|}{} \\
\hline
\end{tabular}


Table 3.

Does SPI Granger-cause per capita GDP Growth?

(Endogenous variable is $\Delta \mathrm{GDP}_{\mathrm{t}}$ )

\begin{tabular}{||l|c|c|}
\hline & $\Delta$ GDP $_{\mathrm{t}-1}$ & $\Delta$ LBSPI $_{\mathrm{t}-1}$ \\
\hline All LDCs & .1020 & -.1603 \\
& $(1.48)$ & $(-.73)$ \\
\hline Asia & .310948 & .3553 \\
& $(1.46)$ & $(.83)$ \\
\hline Latin America & -.1744 & .098049 \\
& $(-1.28)$ & $(.34)$ \\
\hline Middle East \& North & .1701 & -.2473 \\
Africa & $(1.32)$ & $(-.35)$ \\
\hline Sub-Saharan Africa & .1192 & $-.8550 *$ \\
& $(1.02)$ & $(-1.96)$ \\
\hline
\end{tabular}

(Endogenous variable is $\Delta \mathrm{GDP}_{\mathrm{t}}$ )

\begin{tabular}{||l|c|c||}
\hline & $\Delta$ GDP $_{\mathrm{t}-1}$ & $\Delta$ UBSPI $_{\mathrm{t}-1}$ \\
\hline All LDCs & .0880 & .3629 \\
& $(.90)$ & $(1.56)$ \\
\hline Asia & .3595 & -.3424 \\
& $(1.25)$ & $(-.8288)$ \\
\hline Latin America & -.0560 & .5528 \\
& $(-.31)$ & $(1.21)$ \\
\hline Middle East \& North & .1687 & 1.6020 \\
Africa & $(.75)$ & $(1.83)$ \\
\hline Sub-Saharan Africa & .1082 & .0494 \\
& $(.77)$ & $(.15)$ \\
\hline Notes: All variables are in first-differences ( $\Delta)$, five-year averages, between 1960-1995, and t- \\
\hline statistics are in parenthesis. Instrumental variables estimates shown (Anderson-Hsiao-Arellano). \\
LBSPI is lower-bound SPI, UBSPI is upper bound SPI, and GDP is the OLS per capita GDP \\
Growth Rate. \\
* Statistically significant at the 10 percent level. \\
** Statistically significant at the 5 percent level. \\
*** Statistically significant at the 1 percent level.
\end{tabular}


Table 4.

Does per capita GDP Growth Granger-cause SPI?

(Endogenous variable is lower-bound $\Delta \mathrm{LBSPI}_{\mathrm{t}}$ )

\begin{tabular}{||l|c|c|}
\hline & $\Delta$ LBSPI $_{\mathrm{t}-1}$ & $\Delta$ GDP $_{\mathrm{t}-1}$ \\
\hline All LDCs & $.5434 * * *$ & -.0036 \\
& $(4.48)$ & $(-.33)$ \\
\hline Asia & $.8702 * *$ & -.0153 \\
& $(2.55)$ & $(-.35)$ \\
\hline Latin America & $.5818 * * *$ & .0513 \\
& $(2.99)$ & $(1.61)$ \\
\hline Middle East \& North & .0159 & -.0014 \\
Africa & $(.10) \quad-.13)$ \\
\hline Sub-Saharan Africa & $.6208 * * *$ & -.0276 \\
& $(2.78)$ & $(-1.54)$ \\
\hline
\end{tabular}

(Endogenous variable is upper-bound $\Delta \mathrm{UBSPI}_{\mathrm{t}}$ )

\begin{tabular}{||l|c|c||}
\hline & $\Delta$ UBSPI $_{\mathrm{t}-1}$ & $\Delta \mathrm{GDP}_{\mathrm{t}-1}$ \\
\hline All LDCs & .1773 & -.0021 \\
& $(1.59)$ & $(-.11)$ \\
\hline Asia & .3255 & -.0736 \\
& $(.87)$ & $(-.88)$ \\
\hline Latin America & .0776 & .0673 \\
& $(.34)$ & $(1.56)$ \\
\hline Middle East \& North & .1854 & .0057 \\
Africa & $(1.29)$ & $(.19)$ \\
\hline Sub-Saharan Africa & .2195 & -.0331 \\
& $(1.07)$ & $(-1.08)$ \\
\hline Notes: All variables are in first-differences ( $\Delta)$, five-year averages, between 1960-1995, and t- \\
Statistics are in parenthesis. Instrumental variables estimates shown (Anderson-Hsiao-Arellano). \\
LBSPI is lower-bound SPI, UBSPI is upper bound SPI, and GDP is the OLS per capita GDP \\
Growth Rate. \\
* Statistically significant at the 10 percent level. \\
** Statistically significant at the 5 percent level. \\
*** Statistically significant at the 1 percent level. \\
\hline
\end{tabular}


Table 5.

Controlling for institutions and initial income, does SPI Granger-cause per capita GDP growth?

(Endogenous variable is $\Delta \mathrm{GDP}_{\mathrm{t}}$ )

\begin{tabular}{||l|c|c|c|c|}
\hline & $\Delta \mathrm{GDP}_{\mathrm{t}-1}$ & $\Delta$ LBSPI $_{\mathrm{t}-1}$ & $\Delta$ LEGEF $_{\mathrm{t}-1}$ & $\Delta \mathrm{GDP0}_{\mathrm{t}-1}$ \\
\hline All LDCs & $\begin{array}{c}-.099901 \\
(-.793022)\end{array}$ & $\begin{array}{c}-.127939 \\
(-.579914)\end{array}$ & $\begin{array}{c}-.310724 \\
(-.657877)\end{array}$ & $\begin{array}{c}-.000694 \\
(-.843915)\end{array}$ \\
\hline Asia & $\begin{array}{l}.217832 \\
(1.23315)\end{array}$ & $\begin{array}{c}.025806 \\
(.060398)\end{array}$ & $\begin{array}{c}.308899 \\
(.390275)\end{array}$ & $\begin{array}{c}-.001467 \\
(-1.477620\end{array}$ \\
\hline Latin America & $-.411040 *$ & -.040021 & -.575221 & $-.002343^{*}$ \\
& $(-1.93362)$ & $(-.128262)$ & $(-.888192)$ & $(-1.67330)$ \\
\hline Middle East \& North & .260669 & -.285469 & 1.63488 & .001697 \\
Africa & $(.624948)$ & $(-.334162)$ & $(.756562)$ & $(.654005)$ \\
\hline Sub-Saharan Africa & -.049150 & -.460715 & -1.23415 & -.000452 \\
& $(-.236183)$ & $(-.973416)$ & $(-1.34210)$ & $(-.245089)$ \\
\hline
\end{tabular}

(Endogenous variable is $\Delta \mathrm{GDP}_{\mathrm{t}}$ )

\begin{tabular}{|c|c|c|c|c|}
\hline & $\Delta \mathrm{GDP}_{\mathrm{t}-1}$ & $\Delta \mathrm{UBSPI}_{\mathrm{t}-1}$ & $\Delta \mathrm{LEGEF}_{\mathrm{t}-1}$ & $\Delta \mathrm{GDPO}_{\mathrm{t}-1}$ \\
\hline All LDCs & $\begin{array}{l}-.272948^{*} \\
(-1.84204) \\
\end{array}$ & $\begin{array}{c}.219158 \\
(.932017) \\
\end{array}$ & $\begin{array}{r}.-.524852 \\
(-.886105) \\
\end{array}$ & $\begin{array}{l}-.001768^{*} \\
(-1.89236) \\
\end{array}$ \\
\hline Asia & $\begin{array}{l}.243242 \\
(.977085) \\
\end{array}$ & $\begin{array}{c}.018053 \\
(.044311) \\
\end{array}$ & $\begin{array}{c}-.654817 \\
(-.620857) \\
\end{array}$ & $\begin{array}{l}-.001444 \\
(-1.42580) \\
\end{array}$ \\
\hline Latin America & $\begin{array}{c}-.848040 * * * \\
(-2.68874) \\
\end{array}$ & $\begin{array}{c}-.256357 \\
(-.502485) \\
\end{array}$ & $\begin{array}{c}-1.02379 \\
(-1.14927) \\
\end{array}$ & $\begin{array}{c}-.006313 * * * \\
(-3.14233) \\
\end{array}$ \\
\hline $\begin{array}{l}\text { Middle East \& North } \\
\text { Africa }\end{array}$ & $\begin{array}{c}.341391 \\
(.593774) \\
\end{array}$ & $\begin{array}{c}1.82170 \\
(1.33377) \\
\end{array}$ & $\begin{array}{r}2.28172 \\
(.867335) \\
\end{array}$ & $\begin{array}{c}.002853 \\
(.814455) \\
\end{array}$ \\
\hline Sub-Saharan Africa & $\begin{array}{l}-.215917 \\
(-1.05915) \\
\end{array}$ & $\begin{array}{c}.075596 \\
(.229527) \\
\end{array}$ & $\begin{array}{c}-1.19105 \\
(-1.14784)\end{array}$ & $\begin{array}{l}-.001621 \\
(-.924678) \\
\end{array}$ \\
\hline \multicolumn{5}{|c|}{$\begin{array}{l}\text { Notes: All variables are in first-differences ( } \triangle \text { ), five-year averages, between 1960-1995, and t- } \\
\text { statistics are in parenthesis. Instrumental variables estimates shown (Anderson-Hsiao-Arellano). } \\
\text { LBSPI is lower-bound SPI, UBSPI is upper-bound SPI, GDP is the OLS per capita GDP Growth } \\
\text { Rate, LEGEF is an index of legislative effectiveness (institutional development), and GDPO is level } \\
\text { of initial per capita income. } \\
* \text { Statistically significant at the } 10 \text { percent level. } \\
\text { ** Statistically significant at the } 5 \text { percent level. } \\
* * * \text { Statistically significant at the } 1 \text { percent level. }\end{array}$} \\
\hline
\end{tabular}


Table 6.

Controlling for institutions and initial income, does GDP per capita growth Granger-cause SPI?

(Endogenous variable is $\Delta \mathrm{LBSPI}_{\mathrm{t}}$ )

\begin{tabular}{||l|c|c|c|c||}
\hline & $\Delta$ LBSPI $_{\mathrm{t}-1}$ & $\Delta \mathrm{GDP}_{\mathrm{t}-1}$ & $\Delta \mathrm{LEGEF}_{\mathrm{t}-1}$ & $\Delta \mathrm{GDPO}_{\mathrm{t}-1}$ \\
\hline All LDCs & $.241667 * *$ & .001460 & $-1.21630 * * *$ & -.000082 \\
& $(2.51915)$ & $(.106914)$ & $(-12.6326)$ & $(-.604501)$ \\
\hline Asia & .212834 & .006572 & $-1.23008 * * *$ & -.000027 \\
& $(.948019)$ & $(.210992)$ & $(-6.44086)$ & $(-.114898)$ \\
\hline Latin America & .197626 & $.070435 * *$ & $-1.51822 * * *$ & .000166 \\
& $(1.27543)$ & $(2.14353)$ & $(-8.82059)$ & $(.541064)$ \\
\hline Middle East \& North & -.012567 & -.036122 & $-.542633 * *$ & $-.000476 *$ \\
Africa & $(-.066910)$ & $(-1.19802)$ & $(-2.19795)$ & $(-1.89664)$ \\
\hline Sub-Saharan Africa & $.490306 * * *$ & -.029438 & $-1.07150 * * *$ & -.000153 \\
& $(2.73276)$ & $(-1.38838)$ & $(-6.23833)$ & $(-.601373)$ \\
\hline
\end{tabular}

(Endogenous variable is $\Delta \mathrm{UBSPI}_{\mathrm{t}}$ )

\begin{tabular}{||l|c|c|c|c||}
\hline & $\Delta$ UBSPI $_{\mathrm{t}-1}$ & $\Delta \mathrm{GDP}_{\mathrm{t}-1}$ & $\Delta$ LEGEF $_{\mathrm{t}-1}$ & $\Delta \mathrm{GDP0}_{\mathrm{t}-1}$ \\
\hline All LDCs & .023900 & -.005830 & $-.796847 * * *$ \\
& $(.238744)$ & $(-.284849)$ & $(-4.98379)$ & -.000130 \\
\hline Asia & .170841 & -.065159 & $-1.05159 * *$ & -.000046 \\
& $(.536400)$ & $(-.858917)$ & $(-2.32080)$ & $(-.104656)$ \\
\hline Latin America & -.034635 & .068619 & $-.727868 * * *$ & -.000053 \\
& $(-.162371)$ & $(1.45854)$ & $(-2.91017)$ & $(-.123021)$ \\
\hline Middle East \& North & .047810 & -.019980 & -.471677 & -.000257 \\
Africa & $(.280701)$ & $(-.477918)$ & $(-1.48426)$ & $(-.774756)$ \\
\hline Sub-Saharan Africa & .003319 & -.029328 & $-1.07179 * * *$ & -.000064 \\
& $(.019613)$ & $(-.894690)$ & $(-3.39197)$ & $(-.158291)$ \\
\hline
\end{tabular}

Notes: All variables are in first-differences $(\Delta)$, five-year averages, between 1960-1995, and tstatistics are in parenthesis. Instrumental variables estimates shown (Anderson-Hsiao-Arellano). $\angle B S P I$ is lower-bound SPI, UBSPI is upper-bound SPI, GDP is the OLS per capita GDP Growth Rate, $L E G E F$ is an index of legislative effectiveness (institutional development), and GDPO is level of initial per capita income.

* Statistically significant at the 10 percent level.

** Statistically significant at the 5 percent level.

*** Statistically significant at the 1 percent level. 


\section{APPENDIX TABLES}

\begin{tabular}{|c|c|c|c|}
\hline \multicolumn{4}{|c|}{$\begin{array}{c}\text { Table A1. } \\
\text { Controlling for POPULATION GROWTH, } \\
\text { does SPI Granger cause Growth? } \\
\text { (Endogenous variable is } \Delta \mathrm{GDP}_{\mathrm{t}} \text { ) }\end{array}$} \\
\hline & $\Delta \mathrm{GDP}_{\mathrm{t}-1}$ & $\Delta \mathrm{UBSPI}_{\mathrm{t}-1}$ & $\Delta \mathrm{POP}_{\mathrm{t}-1}$ \\
\hline All LDCs & $\begin{array}{c}.063713 \\
(.673218) \\
\end{array}$ & $\begin{array}{c}.333251 \\
(1.45867) \\
\end{array}$ & $\begin{array}{c}-.996263 * * \\
(-2.55773) \\
\end{array}$ \\
\hline Asia & $\begin{array}{c}.347481 \\
(1.21911)\end{array}$ & $\begin{array}{c}-.320292 \\
(-.764315)\end{array}$ & $\begin{array}{c}.364514 \\
(.253842)\end{array}$ \\
\hline Latin America & $\begin{array}{l}-.048101 \\
(-.263422) \\
\end{array}$ & $\begin{array}{l}.558470 \\
(1.21246) \\
\end{array}$ & $\begin{array}{l}-.096387 \\
(-.060595) \\
\end{array}$ \\
\hline $\begin{array}{l}\text { Middle East \& North } \\
\text { Africa }\end{array}$ & $\begin{array}{r}.120133 \\
(.573351) \\
\end{array}$ & $\begin{array}{c}1.38320 \\
(1.62525) \\
\end{array}$ & $\begin{array}{l}-1.13134 \\
(-1.46858) \\
\end{array}$ \\
\hline Sub-Saharan Africa & $\begin{array}{l}.071367 \\
(.525525) \\
\end{array}$ & $\begin{array}{l}.064364 \\
(.199753) \\
\end{array}$ & $\begin{array}{l}-.928208 \\
(-1.56678) \\
\end{array}$ \\
\hline \multicolumn{4}{|c|}{ (Endogenous variable is $\Delta \mathrm{GDP}_{\mathrm{t}}$ ) } \\
\hline & $\Delta \mathrm{GDP}_{\mathrm{t}-1}$ & $\Delta$ LBSPI $_{t-1}$ & $\Delta \mathrm{POP}_{\mathrm{t}-1}$ \\
\hline All LDCs & $\begin{array}{l}.1077099 \\
(1.33395) \\
\end{array}$ & $\begin{array}{l}-.154811 \\
(-.516021)\end{array}$ & $\begin{array}{l}-.977435^{* *} * \\
(-2.47625)\end{array}$ \\
\hline Asia & $\begin{array}{c}.290755 \\
(1.08038) \\
\end{array}$ & $\begin{array}{l}-.115740 \\
(-.211145) \\
\end{array}$ & $\begin{array}{c}.877745 \\
(.606567) \\
\end{array}$ \\
\hline Latin America & $\begin{array}{l}-.070321 \\
(-.392847) \\
\end{array}$ & $\begin{array}{l}.336406 \\
(.763578) \\
\end{array}$ & $\begin{array}{l}.002995 \\
(.001888) \\
\end{array}$ \\
\hline $\begin{array}{l}\text { Middle East \& North } \\
\text { Africa }\end{array}$ & $\begin{array}{c}.171345 \\
(1.12078) \\
\end{array}$ & $\begin{array}{c}-.018353 \\
(-.012682) \\
\end{array}$ & $\begin{array}{l}-1.34555^{*} \\
(-1.70763) \\
\end{array}$ \\
\hline Sub-Saharan Africa & $\begin{array}{l}.104306 \\
(.807724) \\
\end{array}$ & $\begin{array}{l}-800627^{*} \\
(-1.66537) \\
\end{array}$ & $\begin{array}{l}-.743341 \\
(-1.25646) \\
\end{array}$ \\
\hline \multicolumn{4}{|c|}{$\begin{array}{l}\text { Notes: All variables are in first-differences }(\Delta) \text {; five-year averages, between 1960-1995, an } \\
\text { t-statistics are in parentheses. Instrumental variables estimates shown (Anderson-Hsiao } \\
\text { Arellano). } \\
\text { * Statistically significant at the } 10 \text { percent level. } \\
\text { ** Statistically significant at the } 5 \text { percent level. } \\
\text { *** Statistically significant at the } 1 \text { percent level. }\end{array}$} \\
\hline
\end{tabular}


Table A2.

Controlling for POPULATION GROWTH , does growth Granger cause SPI?

(Endogenous variable is $\Delta \mathrm{UBSPI}_{\mathrm{t}}$ )

\begin{tabular}{|c|c|c|c|}
\hline & $\Delta \mathrm{UBSPI}_{\mathrm{t}-1}$ & $\Delta \mathrm{GDP}_{\mathrm{t}-1}$ & $\Delta \mathrm{POP}_{\mathrm{t}-1}$ \\
\hline All LDCs & $\begin{array}{c}.177096 \\
(1.58257) \\
\end{array}$ & $\begin{array}{l}-.002293 \\
(-.119629) \\
\end{array}$ & $\begin{array}{l}-.019691 \\
(-.125354) \\
\end{array}$ \\
\hline Asia & $\begin{array}{c}.357684 \\
(.877997)\end{array}$ & $\begin{array}{l}-.050176 \\
(-.546921)\end{array}$ & $\begin{array}{c}.492166 \\
(.674980)\end{array}$ \\
\hline Latin America & $\begin{array}{c}.070654 \\
(.313628) \\
\end{array}$ & $\begin{array}{c}.064832 \\
(1.50734) \\
\end{array}$ & $\begin{array}{l}-.501138 \\
(-1.04395) \\
\end{array}$ \\
\hline $\begin{array}{l}\text { Middle East \& North } \\
\text { Africa }\end{array}$ & $\begin{array}{c}.227904 \\
(1.44434) \\
\end{array}$ & $\begin{array}{c}.010491 \\
(.340054) \\
\end{array}$ & $\begin{array}{c}.313509 \\
(1.128160 \\
\end{array}$ \\
\hline Sub-Saharan Africa & $\begin{array}{c}.235191 \\
(1.12014)\end{array}$ & $\begin{array}{l}-.034555 \\
(-1.10513)\end{array}$ & $\begin{array}{l}-.128745 \\
(-.563433) \\
\end{array}$ \\
\hline
\end{tabular}

(Endogenous variable is $\Delta \mathrm{LBSPI}_{\mathrm{t}}$ )

\begin{tabular}{||l|c|c|c|}
\hline & $\Delta$ LBSPI $_{\mathrm{t}-1}$ & $\Delta \mathrm{GDP}_{\mathrm{t}-1}$ & $\Delta \mathrm{POP}_{\mathrm{t}-1}$ \\
\hline All LDCs & $\begin{array}{c}.727157 * * * \\
(4.48790)\end{array}$ & $\begin{array}{c}-.005373 \\
(-.460717)\end{array}$ & $\begin{array}{c}-.040605 \\
(-.451696)\end{array}$ \\
\hline Asia & $\begin{array}{l}1.15754 * * \\
(2.42053)\end{array}$ & $\begin{array}{c}-.034005 \\
(-.624177)\end{array}$ & $\begin{array}{c}-.436315 \\
(-.796154)\end{array}$ \\
\hline Latin America & $.747953 * * *$ & $.060699 *$ & $.781040 *$ \\
& $(3.12212)$ & $(1.75235)$ & $(1.68018)$ \\
\hline Middle East \& North & $.987216^{*} *$ & -.007426 & -.077700 \\
Africa & $(2.00831)$ & $(-.555842)$ & $(-.889653)$ \\
\hline Sub-Saharan Africa & $.502235^{*} *$ & -.026458 & -.093244 \\
& $(2.22661)$ & $(-1.47059)$ & $(-.743186)$ \\
\hline
\end{tabular}

Notes: All variables are in first-differences $(\Delta)$; five-year averages, between 1960-1995, and t-statistics are in parentheses. Instrumental variables estimates shown (Anderson-HsiaoArellano).

* Statistically significant at the 10 percent level.

** Statistically significant at the 5 percent level.

*** Statistically significant at the 1 percent level. 
Table A3.

Controlling for GROWTH OF TRADE PARTNERS, does UBSPI Granger cause Growth?

(Endogenous variable is $\Delta \mathrm{GDP}_{\mathrm{t}}$ )

\begin{tabular}{||l|c|c|c|}
\hline & $\Delta$ GDP $_{\mathrm{t}-1}$ & $\Delta \mathrm{UBSPI}_{\mathrm{t}-1}$ & $\Delta \mathrm{GTR}_{\mathrm{t}-1}$ \\
\hline All LDCs & .027706 & .319729 & -.277978 \\
& $(.260707)$ & $(1.38067)$ & $(-1.08602)$ \\
\hline Asia & .514409 & -.433201 & $-.902744 * *$ \\
& $(1.44240)$ & $(-1.00014)$ & $(-2.13731)$ \\
\hline Latin America & -.181755 & .469721 & -.361112 \\
& $(-1.01994)$ & $(1.07862)$ & $(-.627208)$ \\
\hline Middle East \& North & .103222 & $1.52685 *$ & .786441 \\
Africa & $(.443000)$ & $(1.69143)$ & $(1.10039)$ \\
\hline Sub-Saharan Africa & .037149 & .033208 & -.537624 \\
& $(.241965)$ & $(.103939)$ & $(-1.37845)$ \\
\hline
\end{tabular}

(Endogenous variable is $\Delta \mathrm{GDP}_{\mathrm{t}}$ )

\begin{tabular}{||l|c|c|c|}
\hline & $\Delta \mathrm{GDP}_{\mathrm{t}-1}$ & $\Delta$ LBSPI $_{\mathrm{t}-1}$ & $\Delta \mathrm{GTR}_{\mathrm{t}-1}$ \\
\hline All LDCs & $\begin{array}{c}.090733 \\
(1.01752)\end{array}$ & $\begin{array}{c}-.139800 \\
(-.458321)\end{array}$ & $\begin{array}{c}-.317800 \\
(-1.25765)\end{array}$ \\
\hline Asia & .339162 & -.126568 & $-.789257^{*} *$ \\
& $(1.14546)$ & $(-.238707)$ & $(-2.04404)$ \\
\hline Latin America & -.222673 & .354821 & -.361688 \\
& $(-1.29501)$ & $(.845274)$ & $(-.627232)$ \\
\hline Middle East \& North & .146200 & -.350853 & .690579 \\
Africa & $(.871692)$ & $(-.235615)$ & $(.927054)$ \\
\hline Sub-Saharan Africa & .077057 & $-.879801 *$ & $-.628217 *$ \\
& $(.528958)$ & $(-1.80158)$ & $(-1.73434)$ \\
\hline
\end{tabular}

Notes: All variables are in first-differences $(\Delta)$; five-year averages, between 1960-1995, and t-statistics are in parentheses. Instrumental variables estimates shown (Anderson-HsiaoArellano).

* Statistically significant at the 10 percent level.

** Statistically significant at the 5 percent level.

*** Statistically significant at the 1 percent level. 
Table A4.

Controlling for GROWTH OF TRADE PARTNERS , does growth Granger cause UBSPI?

(Endogenous variable is $\triangle \mathrm{UBSPI}_{\mathrm{t}}$ )

\begin{tabular}{||l|c|c|c|}
\hline & $\Delta$ UBSPI $_{\mathrm{t}-1}$ & $\Delta \mathrm{GDP}_{\mathrm{t}-1}$ & $\Delta \mathrm{GTR}_{\mathrm{t}-1}$ \\
\hline All LDCs & .168124 & .002781 & -.098260 \\
& $(1.42441$ & $(.129847)$ & $(-1.01434)$ \\
\hline Asia & .342873 & -.076887 & .048993 \\
& $(.830166)$ & $(-.763641)$ & $(.228379)$ \\
\hline Latin America & .059153 & .062023 & -.141492 \\
& $(.259548)$ & $(1.38413)$ & $(-.502934)$ \\
\hline Middle East \& North & .172882 & .007879 & .101710 \\
Africa & $(1.10923)$ & $(.238707)$ & $(.590362)$ \\
\hline Sub-Saharan Africa & .244716 & -.029368 & -.237864 \\
& $(1.01520)$ & $(.768695)$ & $(-1.21281)$ \\
\hline
\end{tabular}

(Endogenous variable is $\Delta \mathrm{LBSPI}_{\mathrm{t}}$ )

\begin{tabular}{|c|c|c|c|}
\hline & $\Delta$ LBSPI $_{\mathrm{t}-1}$ & $\Delta \mathrm{GDP}_{\mathrm{t}-1}$ & $\Delta \mathrm{GTR}_{\mathrm{t}-1}$ \\
\hline All LDCs & $\begin{array}{c}.730082 * * * \\
(4.54081)\end{array}$ & $\begin{array}{l}-.004363 \\
(-.336497)\end{array}$ & $\begin{array}{c}.062273 \\
(1.25365)\end{array}$ \\
\hline Asia & $\begin{array}{l}1.12818 * * \\
(2.50427)\end{array}$ & $\begin{array}{l}-.037148 \\
(-.653014)\end{array}$ & $\begin{array}{l}.111635 \\
(.881780)\end{array}$ \\
\hline Latin America & $\begin{array}{c}.765661 * * * \\
(3.16806)\end{array}$ & $\begin{array}{c}.054805 \\
(1.46851) \\
\end{array}$ & $\begin{array}{l}.094215 \\
(.572528) \\
\end{array}$ \\
\hline $\begin{array}{l}\text { Middle East \& North } \\
\text { Africa }\end{array}$ & $\begin{array}{l}.968833 * * \\
(1.99367)\end{array}$ & $\begin{array}{l}-.007356 \\
(-.525418)\end{array}$ & $\begin{array}{l}-.006187 \\
(-.087783)\end{array}$ \\
\hline Sub-Saharan Africa & $\begin{array}{l}.538116 * * \\
(2.20668)\end{array}$ & $\begin{array}{l}-.029093 \\
(-1.29039)\end{array}$ & $\begin{array}{c}.069778 \\
(1.01961)\end{array}$ \\
\hline
\end{tabular}

Notes: All variables are in first-differences $(\Delta)$; five-year averages, between 1960-1995, and t-statistics are in parentheses. Instrumental variables estimates shown (Anderson-HsiaoArellano).

* Statistically significant at the 10 percent level.

** Statistically significant at the 5 percent level.

*** Statistically significant at the 1 percent level. 
Table A5.

Controlling for TERMS OF TRADE, does SPI Granger cause Growth?

(Endogenous variable is $\Delta \mathrm{GDP}_{\mathrm{t}}$ )

\begin{tabular}{||l|c|c|c|}
\hline & $\Delta \Delta \mathrm{GDP}_{\mathrm{t}-1}$ & $\Delta \mathrm{UBSPI}_{\mathrm{t}-1}$ & $\Delta$ TRADE $_{\mathrm{t}-1}$ \\
\hline All LDCs & -.018488 & .157844 & $-13.8265^{*}$ \\
& $(-.179112)$ & $(.565360)$ & $(-1.66314)$ \\
\hline Asia & .559613 & $-1.03075^{*}$ & -46.1092 \\
& $(1.13233)$ & $(-1.90834)$ & $(-1.60947)$ \\
\hline Latin America & .001457 & .865198 & $-90.9842 * *$ \\
& $(.006833)$ & $(1.55346)$ & $(-2.55452)$ \\
\hline Middle East \& North & -.060317 & 1.03243 & 8.66788 \\
Africa & $(-.258443)$ & $(.769931)$ & $(.337650)$ \\
\hline Sub-Saharan Africa & .120246 & -.088595 & -12.9814 \\
& $(.734215)$ & $(-.202830)$ & $(-1.19894)$ \\
\hline
\end{tabular}

(Endogenous variable is $\Delta \mathrm{GDP}_{\mathrm{t}}$ )

\begin{tabular}{||l|c|c|c|}
\hline & $\Delta$ GDP $_{\mathrm{t}-1}$ & $\Delta$ LBSPI $_{\mathrm{t}-1}$ & $\Delta \mathrm{TRADE}_{\mathrm{t}-1}$ \\
\hline All LDCs & \begin{tabular}{c|c}
.058502 \\
$(.676804)$
\end{tabular} & $\begin{array}{c}.018173 \\
(.049225)\end{array}$ & $\begin{array}{l}-16.1476^{*} \\
(-1.86851)\end{array}$ \\
\hline Asia & .394451 & .790993 & -45.4362 \\
& $(.985616)$ & $(.942705)$ & $(-1.47553)$ \\
\hline Latin America & -.065674 & .548343 & $-81.8608^{* *}$ \\
& $(-.331421)$ & $(1.06997)$ & $(-2.40753)$ \\
\hline Middle East \& North & .107492 & -1.64159 & 15.0812 \\
Africa & $(.698454)$ & $(-.560102)$ & $(.541614)$ \\
\hline Sub-Saharan Africa & .158013 & -.950590 & -16.5602 \\
& $(.956214)$ & $(-1.36600)$ & $(-1.50938)$ \\
\hline
\end{tabular}

Notes: All variables are in first-differences $(\Delta)$; five-year averages, between 1960-1995, and t-statistics are in parentheses. Instrumental variables estimates shown (Anderson-HsiaoArellano).

* Statistically significant at the 10 percent level.

** Statistically significant at the 5 percent level.

*** Statistically significant at the 1 percent level. 
Table A6.

Controlling for TERMS OF TRADE, does growth Granger cause UBSPI?

(Endogenous variable is $\Delta \mathrm{UBSPI}_{\mathrm{t}}$ )

\begin{tabular}{||l|c|c|c|}
\hline & $\Delta$ UBSPI $_{\mathrm{t}-1}$ & $\Delta \mathrm{GDP}_{\mathrm{t}-1}$ & $\Delta$ TRADE $_{\mathrm{t}-1}$ \\
\hline All LDCs & $\begin{array}{c}.080099 \\
(.558266)\end{array}$ & $\begin{array}{c}-.008692 \\
(-.398951)\end{array}$ & $\begin{array}{c}-.935119 \\
(-.364147)\end{array}$ \\
\hline Asia & .265709 & -.001380 & $17.0913 *$ \\
& $(.646096)$ & $(-.013287)$ & $(1.95409)$ \\
\hline Latin America & .180841 & .090800 & -12.1262 \\
& $(.629474)$ & $(1.51321)$ & $(-1.17756)$ \\
\hline Middle East \& North & .236310 & .007069 & -1.11948 \\
Africa & $(1.12685)$ & $(.217414)$ & $(-.329785)$ \\
\hline Sub-Saharan Africa & -.031384 & -.044168 & -.917071 \\
& $(-.139568)$ & $(-1.37238)$ & $(-.257331)$ \\
\hline
\end{tabular}

(Endogenous variable is $\Delta \mathrm{LBSPI}_{\mathrm{t}}$ )

\begin{tabular}{||l|c|c|c|}
\hline & $\Delta$ LBSPI $_{\mathrm{t}-1}$ & $\Delta$ GDP $_{\mathrm{t}-1}$ & $\Delta$ TRADE $_{\mathrm{t}-1}$ \\
\hline All LDCs & $\begin{array}{c}.698154 * * * \\
(3.78818)\end{array}$ & $\begin{array}{c}-.005652 \\
(-.418939)\end{array}$ & $\begin{array}{c}2.60659 \\
(1.43548)\end{array}$ \\
\hline Asia & $1.13936^{* *}$ & .027353 & $-24.9810 * *$ \\
& $(1.98261)$ & $(.409489)$ & $(-2.01537)$ \\
\hline Latin America & $.693441^{* * *}$ & .053226 & 2.36615 \\
& $(2.74535)$ & $(1.38417)$ & $(.347874)$ \\
\hline Middle East \& North & .273076 & -.000573 & -1.92029 \\
Africa & $(.562626)$ & $(-.048486)$ & $(-.741188)$ \\
\hline Sub-Saharan Africa & $.525919 *$ & $-.038697 *$ & $4.95784 * *$ \\
& $(1.91550)$ & $(-1.66179)$ & $(2.51766)$ \\
\hline
\end{tabular}

Notes: All variables are in first-differences $(\Delta)$; five-year averages, between 1960-1995, and t-statistics are in parentheses. Instrumental variables estimates shown (Anderson-HsiaoArellano).

* Statistically significant at the 10 percent level.

** Statistically significant at the 5 percent level.

*** Statistically significant at the 1 percent level. 


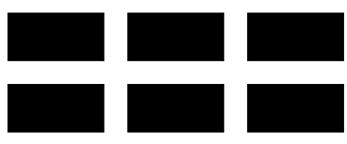

THE WILLIAM DAVIDSON INSTITUTE

AT THE UNIVERSITY OF MICHIGAN BUSINESSSCHOOL

\section{DAVIDSON INSTITUTE WORKING PAPER SERIES}

CURRENT AS OF 8/02/00

\begin{tabular}{|c|c|c|}
\hline Publication & Authors & Date of Paper \\
\hline No. 326 Who is Afraid of Political Instability? & Nauro F. Campos and Jeffrey B. Nugent & July 2000 \\
\hline $\begin{array}{l}\text { No. } 325 \text { Business Groups, the Financial } \\
\text { Market and Modernization }\end{array}$ & Raja Kali & June 2000 \\
\hline $\begin{array}{l}\text { No. } 324 \text { Restructuring with What Success? A } \\
\text { Case Study of Russian Firms }\end{array}$ & Susan Linz & July 2000 \\
\hline $\begin{array}{l}\text { No. } 323 \text { Priorities and Sequencing in } \\
\text { Privatization: Theory and Evidence from the } \\
\text { Czech Republic }\end{array}$ & $\begin{array}{l}\text { Nandini Gupta, John C. Ham and Jan } \\
\text { Svejnar }\end{array}$ & May 2000 \\
\hline $\begin{array}{l}\text { No. } 322 \text { Liquidity, Volatility, and Equity } \\
\text { Trading Costs Across Countries and Over } \\
\text { Time }\end{array}$ & $\begin{array}{l}\text { Ian Domowitz, Jack Glen and Ananth } \\
\text { Madhavan }\end{array}$ & March 2000 \\
\hline $\begin{array}{l}\text { No. } 321 \text { Equilibrium Wage Arrears: } \\
\text { Institutional Lock-In of Contractual Failure in } \\
\text { Russia }\end{array}$ & John S. Earle and Klara Z. Sabirianova & June 2000 \\
\hline $\begin{array}{l}\text { No. } 320 \text { Rethinking Marketing Programs for } \\
\text { Emerging Markets }\end{array}$ & Niraj Dawar and Amitava Chattopadhyay & June 2000 \\
\hline $\begin{array}{l}\text { No. } 319 \text { Public Finance and Low Equilibria in } \\
\text { Transition Economies; the Role of Institutions }\end{array}$ & Daniel Daianu and Radu Vranceanu & June 2000 \\
\hline $\begin{array}{l}\text { No. } 318 \text { Some Econometric Evidence on the } \\
\text { Effectiveness of Active Labour Market } \\
\text { Programmes in East Germany }\end{array}$ & Martin Eichler and Michael Lechner & June 2000 \\
\hline $\begin{array}{l}\text { No. } 317 \text { A Model of Russia's "Virtual } \\
\text { Economy" }\end{array}$ & R.E Ericson and B.W Ickes & May 2000 \\
\hline $\begin{array}{l}\text { No. } 316 \text { Financial Institutions, Financial } \\
\text { Contagion, and Financial Crises }\end{array}$ & Haizhou Huang and Chenggang $X u$ & March 2000 \\
\hline $\begin{array}{l}\text { No. } 315 \text { Privatization versus Regulation in } \\
\text { Developing Economies: The Case of West } \\
\text { African Banks }\end{array}$ & $\begin{array}{l}\text { Jean Paul Azam, Bruno Biais, and } \\
\text { Magueye Dia }\end{array}$ & February 2000 \\
\hline $\begin{array}{l}\text { No. } 314 \text { Is Life More Risky in the Open? } \\
\text { Household Risk-Coping and the Opening of } \\
\text { China's Labor Markets }\end{array}$ & John Giles & April 2000 \\
\hline $\begin{array}{l}\text { No. } 313 \text { Networks, Migration and Investment: } \\
\text { Insiders and Outsiders in Tirupur's } \\
\text { Production Cluster }\end{array}$ & Abhijit Banerjee and Kaivan Munshi & March 2000 \\
\hline $\begin{array}{l}\text { No. } 312 \text { Computational Analysis of the Impact } \\
\text { on India of the Uruguay Round and the } \\
\text { Forthcoming WTO Trade Negotiations }\end{array}$ & $\begin{array}{l}\text { Rajesh Chadha, Drusilla K. Brown, Alan } \\
\text { V. Deardorff and Robert M. Stern }\end{array}$ & March 2000 \\
\hline $\begin{array}{l}\text { No. } 311 \text { Subsidized Jobs for Unemployed } \\
\text { Workers in Slovakia }\end{array}$ & Jan. C. van Ours & May 2000 \\
\hline $\begin{array}{l}\text { No. } 310 \text { Determinants of Managerial Pay in } \\
\text { the Czech Republic }\end{array}$ & $\begin{array}{l}\text { Tor Eriksson, Jaromir Gottvald and Pavel } \\
\text { Mrazek }\end{array}$ & May 2000 \\
\hline $\begin{array}{l}\text { No. } 309 \text { The Great Human Capital } \\
\text { Reallocation: An Empirical Analysis of } \\
\text { Occupational Mobility in Transitional Russia }\end{array}$ & Klara Z. Sabirianova & May 2000 \\
\hline $\begin{array}{l}\text { No. } 308 \text { Economic Development, Legality, and } \\
\text { the Transplant Effect }\end{array}$ & $\begin{array}{l}\text { Daniel Berkowitz, Katharina Pistor, and } \\
\text { Jean-Francois Richard }\end{array}$ & February 2000 \\
\hline
\end{tabular}

Publications denoted by an asterisk are not available on the Kresge Library webpage (http://www.wdi.bus.umich.edu) 


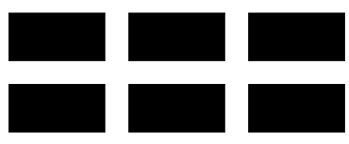

THE WILLIAM DAVIDSON INSTITUTE

AT THE UNIVERSITY OF MICHIGAN BUSINESSSCHOOL

\begin{tabular}{|c|c|c|}
\hline $\begin{array}{l}\text { No. } 307 \text { Community Participation, Teacher } \\
\text { Effort, and Educational Outcome: The Case of } \\
\text { El Salvador's EDUCO Program }\end{array}$ & Yasuyuki Sawada & November 1999 \\
\hline $\begin{array}{l}\text { No. } 306 \text { Gender Wage Gap and Segregation in } \\
\text { Late Transition }\end{array}$ & Stepan Jurajda & May 2000 \\
\hline $\begin{array}{l}\text { No. } 305 \text { The Gender Pay Gap in the } \\
\text { Transition from Communism: Some Empirical } \\
\text { Evidence }\end{array}$ & Andrew Newell and Barry Reilly & May 2000 \\
\hline $\begin{array}{l}\text { No. } 304 \text { Post-Unification Wage Growth in } \\
\text { East Germany }\end{array}$ & Jennifer Hunt & November 1998 \\
\hline $\begin{array}{l}\text { No. } 303 \text { How Does Privatization Affect } \\
\text { Workers? The Case of the Russian Mass } \\
\text { Privatization Program }\end{array}$ & Elizabeth Brainerd & May 2000 \\
\hline $\begin{array}{l}\text { No. } 302 \text { Liability for Past Environmental } \\
\text { Contamination and Privatization }\end{array}$ & Dietrich Earnhart & March 2000 \\
\hline No. 301 Varieties, Jobs and EU Enlargement & Tito Boeri and Joaquim Oliveira Martins & May 2000 \\
\hline No. 300 Employer Size Effects in Russia & Todd Idson & April 2000 \\
\hline $\begin{array}{l}\text { No. } 299 \text { Information Complements, } \\
\text { Substitutes, and Strategic Product Design }\end{array}$ & $\begin{array}{l}\text { Geoffrey G. Parker and Marshall W. Van } \\
\text { Alstyne }\end{array}$ & March 2000 \\
\hline $\begin{array}{l}\text { No. } 298 \text { Markets, Human Capital, and } \\
\text { Inequality: Evidence from Rural China }\end{array}$ & $\begin{array}{l}\text { Dwayne Benjamin, Loren Brandt, Paul } \\
\text { Glewwe, and Li Guo }\end{array}$ & May 2000 \\
\hline $\begin{array}{l}\text { No. } 297 \text { Corporate Governance in the Asian } \\
\text { Financial Crisis }\end{array}$ & $\begin{array}{l}\text { Simon Johnson, Peter Boone, Alasdair } \\
\text { Breach, and Eric Friedman }\end{array}$ & November 1999 \\
\hline $\begin{array}{l}\text { No. } 296 \text { Competition and Firm Performance: } \\
\text { Lessons from Russia }\end{array}$ & J. David Brown and John S. Earle & March 2000 \\
\hline $\begin{array}{l}\text { No. } 295 \text { Wage Determination in Russia: An } \\
\text { Econometric Investigation }\end{array}$ & Peter J. Luke and Mark E. Schaffer & March 2000 \\
\hline $\begin{array}{l}\text { No. 294: Can Banks Promote Enterprise } \\
\text { Restructuring?: Evidence From a Polish } \\
\text { Bank's Experience }\end{array}$ & John P. Bonin and Bozena Leven & March 2000 \\
\hline $\begin{array}{l}\text { No. 293: Why do Governments Sell Privatised } \\
\text { Companies Abroad? }\end{array}$ & $\begin{array}{l}\text { Bernardo Bortolotti, Marcella Fantini and } \\
\text { Carlo Scarpa }\end{array}$ & March 2000 \\
\hline $\begin{array}{l}\text { No. 292: Going Public in Poland: Case-by- } \\
\text { Case Privatizations, Mass Privatization and } \\
\text { Private Sector Initial Public Offerings }\end{array}$ & Wolfgang Aussenegg & December 1999 \\
\hline $\begin{array}{l}\text { No. 291: Institutional Technology and the } \\
\text { Chains of Trust: Capital Markets and } \\
\text { Privatization in Russia and the Czech } \\
\text { Republic }\end{array}$ & Bruce Kogut and Andrew Spicer & March 1999 \\
\hline $\begin{array}{l}\text { No. 290: Banking Crises and Bank Rescues: } \\
\text { The Effect of Reputation }\end{array}$ & Jenny Corbett and Janet Mitchell & January 2000 \\
\hline $\begin{array}{l}\text { No. 289: Do Active Labor Market Policies } \\
\text { Help Unemployed Workers to Find and Keep } \\
\text { Regular Jobs? }\end{array}$ & Jan C. van Ours & February 2000 \\
\hline $\begin{array}{l}\text { No. 288: Consumption Patterns of the New } \\
\text { Elite in Zimbabwe }\end{array}$ & Russell Belk & February 2000 \\
\hline $\begin{array}{l}\text { No. 287: Barter in Transition Economies: } \\
\text { Competing Explanations Confront Ukranian } \\
\text { Data }\end{array}$ & $\begin{array}{l}\text { Dalia Marin, Daniel Kaufmann and } \\
\text { Bogdan Gorochowskij }\end{array}$ & Janиагу 2000 \\
\hline $\begin{array}{l}\text { No. 286: The Quest for Pension Reform: } \\
\text { Poland's Security through Diversity }\end{array}$ & Marek Góra and Michael Rutkowski & January 2000 \\
\hline
\end{tabular}

Publications denoted by an asterisk are not available on the Kresge Library webpage (http://www.wdi.bus.umich.edu) 


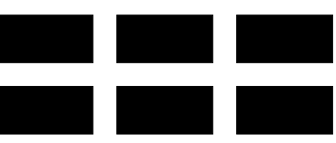

THE WILLIAM DAVIDSON INSTITUTE AT THE UNIVERSITY OF MICHIGAN BUSINESSSCHOOL

\begin{tabular}{|c|c|c|}
\hline $\begin{array}{l}\text { No. 285: Disorganization and Financial } \\
\text { Collapse }\end{array}$ & Dalia Marin and Monika Schnitzer & October 1999 \\
\hline $\begin{array}{l}\text { No. 284: Coordinating Changes in M-form } \\
\text { and U-form Organizations }\end{array}$ & $\begin{array}{l}\text { Yingyi Qian, Gérard Roland and } \\
\text { Chenggang } X u\end{array}$ & May 1999 \\
\hline $\begin{array}{l}\text { No. 283: Why Russian Workers Do Not Move: } \\
\text { Attachment of Workers Through In-Kind } \\
\text { Payments }\end{array}$ & Guido Friebel and Sergei Guriev & October 1999 \\
\hline $\begin{array}{l}\text { No. 282: Lessons From Fiascos in Russian } \\
\text { Corporate Governance }\end{array}$ & Merritt B. Fox and Michael A. Heller & October 1999 \\
\hline $\begin{array}{l}\text { No. 281: Income Distribution and Price } \\
\text { Controls: Targeting a Social Safety Net } \\
\text { During Economic Transition }\end{array}$ & Michael Alexeev and James Leitzel & March 1999 \\
\hline $\begin{array}{l}\text { No. 280: Starting Positions, Reform Speed, } \\
\text { and Economic Outcomes in Transitioning } \\
\text { Economies }\end{array}$ & William Hallagan and Zhang Jun & January 2000 \\
\hline No. 279 : The Value of Prominent Directors & Yoshiro Miwa \& J. Mark Ramseyer & October 1999 \\
\hline No. 278: The System Paradigm & János Kornai & April 1998 \\
\hline $\begin{array}{l}\text { No. 277: The Developmental Consequences of } \\
\text { Foreign Direct Investment in the Transition } \\
\text { from Socialism to Capitalism: The } \\
\text { Performance of Foreign Owned Firms in } \\
\text { Hungary }\end{array}$ & Lawrence Peter King & September 1999 \\
\hline $\begin{array}{l}\text { No. 276: Stability and Disorder: An } \\
\text { Evolutionary Analysis of Russia's Virtual } \\
\text { Economy }\end{array}$ & Clifford Gaddy and Barry W. Ickes & November 1999 \\
\hline $\begin{array}{l}\text { No. 275: Limiting Government Predation } \\
\text { Through Anonymous Banking: A Theory with } \\
\text { Evidence from China. }\end{array}$ & $\begin{array}{l}\text { Chong-En Bai, David D. Li, Yingyi Qian } \\
\text { and Yijiang Wang }\end{array}$ & July 1999 \\
\hline *No. 274: Transition with Labour Supply & Tito Boeri & December 1999 \\
\hline $\begin{array}{l}\text { No. 273: Sectoral Restructuring and Labor } \\
\text { Mobility: A Comparative Look at the Czech } \\
\text { Republic }\end{array}$ & Vit Sorm and Katherine Terrell & November 1999 \\
\hline $\begin{array}{l}\text { *No. 272: Published in: Journal of } \\
\text { Comparative Economics "Returns to Human } \\
\text { Capital Under the Communist Wage Grid and } \\
\text { During the Transition to a Market Economy" } \\
\text { Vol. 27, pp. 33-60 1999. }\end{array}$ & $\begin{array}{l}\text { Daniel Munich, Jan Svejnar and Katherine } \\
\text { Terrell }\end{array}$ & October 1999 \\
\hline $\begin{array}{l}\text { No. 271: Barter in Russia: Liquidity Shortage } \\
\text { Versus Lack of Restructuring }\end{array}$ & Sophie Brana and Mathilde Maurel & June 1999 \\
\hline $\begin{array}{l}\text { No. 270: Tests for Efficient Financial } \\
\text { Intermediation with Application to China }\end{array}$ & Albert Park and Kaja Sehrt & March 1999 \\
\hline $\begin{array}{l}\text { No. 269a: Russian Privatization and } \\
\text { Corporate Governance: What Went Wrong? }\end{array}$ & $\begin{array}{l}\text { Bernard Black, Reinier Kraakman and } \\
\text { Anna Tarassova }\end{array}$ & May 2000 \\
\hline $\begin{array}{l}\text { No. 269: Russian Privatization and Corporate } \\
\text { Governance: What Went Wrong? }\end{array}$ & $\begin{array}{l}\text { Bernard Black, Reinier Kraakman and } \\
\text { Anna Tarassova }\end{array}$ & September 1999 \\
\hline $\begin{array}{l}\text { No. 268: Are Russians Really Ready for } \\
\text { Capitalism? }\end{array}$ & Susan Linz & September 1999 \\
\hline $\begin{array}{l}\text { No. 267: Do Stock Markets Promote } \\
\text { Economic Growth? }\end{array}$ & $\begin{array}{l}\text { Randall K. Filer, Jan Hanousek and Nauro } \\
\text { Campos }\end{array}$ & September 1999 \\
\hline No. 266: Objectivity, Proximity and & Arnoud W.A Boot and Jonathan R. Macey & September 1999 \\
\hline
\end{tabular}

Publications denoted by an asterisk are not available on the Kresge Library webpage (http://www.wdi.bus.umich.edu) 


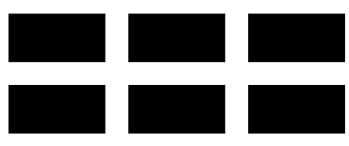

THE WILLIAM DAVIDSON INSTITUTE

AT THE UNIVERSITY OF MICHIGAN BUSINESSSCHOOL

\begin{tabular}{|c|c|c|}
\hline Adaptability in Corporate Governance & & \\
\hline $\begin{array}{l}\text { No. 265: When the Future is not What it Used } \\
\text { to Be: Lessons from the Western European } \\
\text { Experience to Forecasting Education and } \\
\text { Training in Transitional Economies }\end{array}$ & $\begin{array}{l}\text { Nauro F. Campos, Gerard Hughes, Stepan } \\
\text { Jurajda, and Daniel Munich }\end{array}$ & September 1999 \\
\hline $\begin{array}{l}\text { No. 264: The Institutional Foundation of } \\
\text { Foreign-Invested Enterprises (FIEs) in China }\end{array}$ & Yasheng Huang & September 1999 \\
\hline $\begin{array}{l}\text { No. 263: The Changing Corporate } \\
\text { Governance Paradigm: Implications for } \\
\text { Transition and Developing Countries }\end{array}$ & $\begin{array}{l}\text { Erik Berglof and Ernst-Ludwig von } \\
\text { Thadden }\end{array}$ & June 1999 \\
\hline No. 262: Law Enforcement and Transition & Gerard Roland and Thierry Verdier & May 1999 \\
\hline $\begin{array}{l}\text { No. 261: Soft Budget Constraints, Pecuniary } \\
\text { Externality, and the Dual Track System }\end{array}$ & Jiahua Che & June 2000 \\
\hline $\begin{array}{l}\text { No. 260: Missing Market in Labor Quality: } \\
\text { The Role of Quality Markets in Transiton }\end{array}$ & Gary H. Jefferson & July 1999 \\
\hline $\begin{array}{l}\text { No. 259: Do Corporate Global Environmental } \\
\text { Standards in Emerging Markets Create or } \\
\text { Destroy Market Value }\end{array}$ & $\begin{array}{l}\text { Glen Dowell, Stuart Hart and Bernard } \\
\text { Yeung }\end{array}$ & June 1999 \\
\hline $\begin{array}{l}\text { No. 258: Public Training and Outflows from } \\
\text { Unemployment }\end{array}$ & Patrick A. Puhani & June 1999 \\
\hline $\begin{array}{l}\text { No. 257: Ownership Versus Environment: } \\
\text { Why are Public Sector Firms Ineffecient? }\end{array}$ & Ann P. Bartel and Ann E. Harrison & June 1999 \\
\hline $\begin{array}{l}\text { No. 256: Taxation and Evasion in the } \\
\text { Presence of Exortion by Organized Crime }\end{array}$ & $\begin{array}{l}\text { Michael Alexeev, Eckhard Janeba and } \\
\text { Stefan Osborne }\end{array}$ & November 1999 \\
\hline $\begin{array}{l}\text { No. 255: Revisiting Hungary's Bankruptcy } \\
\text { Episode }\end{array}$ & John P. Bonin and Mark E. Schaffer & September 1999 \\
\hline $\begin{array}{l}\text { No. 254: FDI in Emerging Markets: A Home- } \\
\text { Country View }\end{array}$ & Marina v.N Whitman & June 1999 \\
\hline $\begin{array}{l}\text { No. 253: The Asian Financial Crisis: What } \\
\text { Happened, and What is to be Done }\end{array}$ & Jeffrey D. Sachs and Wing Thye Woo & January 1999 \\
\hline $\begin{array}{l}\text { No. 252: Organizational Law as Asset } \\
\text { Partitioning }\end{array}$ & Henry Hansmann and Reinier Kraakman & September 1999 \\
\hline $\begin{array}{l}\text { No. 251: Consumer Behavior Research in } \\
\text { Emerging Consumer Markets: the Case of the } \\
\text { Optimum Stimulation Level in South Africa }\end{array}$ & $\begin{array}{l}\text { Jan-Benedict E. M. Steenkamp and Steven } \\
\text { M. Burgess }\end{array}$ & September 1999 \\
\hline $\begin{array}{l}\text { No. 250: Property Rights Formation and the } \\
\text { Organization of Exchange and Production in } \\
\text { Rural China }\end{array}$ & $\begin{array}{l}\text { Matthew A. Turner, Loren Brandt, and } \\
\text { Scott Rozelle }\end{array}$ & July 1998 \\
\hline $\begin{array}{l}\text { No. 249: Impacts of the Indonesian Economic } \\
\text { Crisis: Price Changes and the Poor }\end{array}$ & $\begin{array}{l}\text { James Levinsohn, Steven Berry, and Jed } \\
\text { Friedman }\end{array}$ & June 1999 \\
\hline $\begin{array}{l}\text { No. 248: Internal Barriers in the Transition of } \\
\text { Enterprises from Central Plan to Market }\end{array}$ & Charalambos Vlachoutsicos & July 1999 \\
\hline $\begin{array}{l}\text { No. 247: Spillovers from Multinationals in } \\
\text { Developing Countries: the Mechanisms at } \\
\text { Work }\end{array}$ & Richard E. Caves & June 1999 \\
\hline $\begin{array}{l}\text { No. 246: Dynamism and Inertia on the } \\
\text { Russian Labour Market: A Model of } \\
\text { Segmentation }\end{array}$ & $\begin{array}{l}\text { Irena Grosfeld, Claudia Senik-Leygonie, } \\
\text { Thierry Verdier, Stanislav Kolenikov and } \\
\text { Elena Paltseva }\end{array}$ & May 1999 \\
\hline $\begin{array}{l}\text { No. 245: Lessons from Bank Privatization in } \\
\text { Central Europe }\end{array}$ & John Bonin and Paul Wachtel & May 1999 \\
\hline No. 244: Nominal-Real Tradeoffs and the & Christian Popa & December 1998 \\
\hline
\end{tabular}

Publications denoted by an asterisk are not available on the Kresge Library webpage (http://www.wdi.bus.umich.edu) 


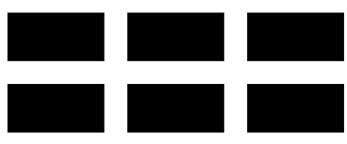

THE WILLIAM DAVIDSON INSTITUTE

AT THE UNIVERSITY OF MICHIGAN BUSINESSSCHOOL

\begin{tabular}{|c|c|c|}
\hline $\begin{array}{l}\text { Effects of Monetary Policy: the Romanian } \\
\text { Experience }\end{array}$ & & \\
\hline $\begin{array}{l}\text { No. 243: Privatization, Political Risk and } \\
\text { Stock Market Development in Emerging } \\
\text { Economies }\end{array}$ & Enrico C. Perotti and Pieter van Oijen & March 1999 \\
\hline $\begin{array}{l}\text { No. 242: Investment Financing in Russian } \\
\text { Financial-Industrial Groups }\end{array}$ & Enrico C. Perotti and Stanislav Gelfer & October 1998 \\
\hline $\begin{array}{l}\text { No. 241: Can governments maintain hard } \\
\text { budget constraints? Bank lending and } \\
\text { financial isolation in Romania }\end{array}$ & $\begin{array}{l}\text { Octavian Carare, Constantijn Claessens, } \\
\text { Enrico C. Perotti }\end{array}$ & January 1999 \\
\hline $\begin{array}{l}\text { No. 240: Democratic Institutions and } \\
\text { Economic Reform: the Polish Case }\end{array}$ & $\begin{array}{l}\text { John E. Jackson, Jacek Klich, and } \\
\text { Krystyna Poznanska }\end{array}$ & April 1998 \\
\hline $\begin{array}{l}\text { No. 239: A Longitudinal Study of IJV } \\
\text { Performance in Eastern Europe }\end{array}$ & Keith D. Brouthers and Gary Bamossy & June 1999 \\
\hline $\begin{array}{l}\text { No. 238: Published in: Journal of Business } \\
\text { Venturing, "Firm Creation and Economic } \\
\text { Transitions" Vol. 14, Iss. 5,6 Sep/Nov 1999, } \\
\text { pp. 427-450. }\end{array}$ & $\begin{array}{l}\text { John E. Jackson, Jacek Klich, Krystyna } \\
\text { Poznanska }\end{array}$ & July 1998 \\
\hline $\begin{array}{l}\text { No. 237: Analysis of Entrepreneurial Attitudes } \\
\text { in Poland }\end{array}$ & $\begin{array}{l}\text { John E. Jackson and Aleksander S. } \\
\text { Marcinkowski }\end{array}$ & March 1997 \\
\hline $\begin{array}{l}\text { No. 236: Investment and Finance in De Novo } \\
\text { Private Firms: Empirical Results from the } \\
\text { Czech Republic, Hungary, and Poland }\end{array}$ & $\begin{array}{l}\text { Andrzej Bratkowski, Irena Grosfeld, Jacek } \\
\text { Rostowski }\end{array}$ & April 1999 \\
\hline $\begin{array}{l}\text { No. 235: Does a Soft Macroeconomic } \\
\text { Environment Induce Restructuring on the } \\
\text { Microeconomic Level during the Transition } \\
\text { Period? Evidence from Investment Behavior } \\
\text { of Czech Enterprises }\end{array}$ & Lubomír Lízal & June 1999 \\
\hline $\begin{array}{l}\text { No. 234: Banking Reform in China: Gradually } \\
\text { Strengthening Pillar or Fragile Reed? }\end{array}$ & John Bonin & June 1999 \\
\hline $\begin{array}{l}\text { No. 233: Theories of Soft Budget Constraints } \\
\text { and the Analysis of Banking Crises }\end{array}$ & Janet Mitchell & March 1999 \\
\hline $\begin{array}{l}\text { No. 232: Unemployment Risk, Precautionary } \\
\text { Savings, and Moonlighting in Russia }\end{array}$ & $\begin{array}{l}\text { Alessandra Guariglia and Byung-Yeon } \\
\text { Kim }\end{array}$ & June 1999 \\
\hline $\begin{array}{l}\text { No. 231: Investing in Turbulent Times: The } \\
\text { Investment Behavior of Polish Firms in the } \\
\text { Transition }\end{array}$ & $\begin{array}{l}\text { Josef C. Brada, Arthur E. King, and Chia- } \\
\text { Ying Ma }\end{array}$ & April 1999 \\
\hline $\begin{array}{l}\text { No. 230: The End of Moderate Inflation in } \\
\text { Three Transition Economies? }\end{array}$ & Josef C. Brada and Ali M. Kutan & April 1999 \\
\hline $\begin{array}{l}\text { No. 229: Back to the Future: The Growth } \\
\text { Prospects of Transition Economies } \\
\text { Reconsidered }\end{array}$ & Nauro F. Campos & April 1999 \\
\hline $\begin{array}{l}\text { No. 228: The Enterprise Isolation Program in } \\
\text { Russia }\end{array}$ & Simeon Djankov & April 1999 \\
\hline $\begin{array}{l}\text { No. 227: Published in: Journal of } \\
\text { Comparative Economics, "Ownership } \\
\text { Concentration and Corporate Performance in } \\
\text { the Czech Republic" 27(3), September 1999, } \\
\text { pp. 498-513. }\end{array}$ & Stijn Claessens and Simeon Djankov & April 1999 \\
\hline $\begin{array}{l}\text { No. 226: Unemployment Benefit Entitlement } \\
\text { and Training Effects in Poland during } \\
\text { Transition }\end{array}$ & Patrick A. Puhani & March 1999 \\
\hline
\end{tabular}

Publications denoted by an asterisk are not available on the Kresge Library webpage (http://www.wdi.bus.umich.edu) 


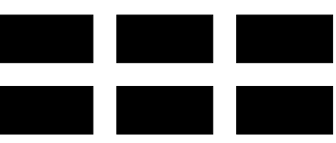

THE WILLIAM DAVIDSON INSTITUTE

AT THE UNIVERSITY OF MICHIGAN BUSINESSSCHOOL

\begin{tabular}{|c|c|c|}
\hline $\begin{array}{l}\text { No. 225: Transition at Whirlpool-Tatramat: } \\
\text { Case Studies }\end{array}$ & Hans Brechbuhl and Sonia Ferencikova & March 1999 \\
\hline $\begin{array}{l}\text { No. 224: Measuring Progress in Transition } \\
\text { and Towards EU Accession: A Comparison of } \\
\text { Manufacturing Firms in Poland, Romania, } \\
\text { and Spain }\end{array}$ & $\begin{array}{l}\text { Wendy Carlin, Saul Estrin, and Mark } \\
\text { Schaffer }\end{array}$ & March 1999 \\
\hline $\begin{array}{l}\text { No. 223: Product Market Competition in } \\
\text { Transition Economies: Increasing Varieties } \\
\text { and Consumer Loyalty }\end{array}$ & Mitsutoshi M. Adachi & March 1999 \\
\hline $\begin{array}{l}\text { No. 222: Opaque Markets and Rapid Growth: } \\
\text { the Superiority of Bank-Centered Financial } \\
\text { Systems for Developing Nations }\end{array}$ & Rodney Wallace & July 1999 \\
\hline $\begin{array}{l}\text { No. 221: Technology Spillovers through } \\
\text { Foreign Direct Investment }\end{array}$ & Yuko Kinoshita & January 1999 \\
\hline $\begin{array}{l}\text { No. 220: Managerial, Expertise and Team } \\
\text { Centered Forms of Organizing: A Cross- } \\
\text { Cultural Exploration of Independence in } \\
\text { Engineering Work }\end{array}$ & Leslie Perlow & January 1999 \\
\hline $\begin{array}{l}\text { No. 219: Household Structure and Labor } \\
\text { Demand in Agriculture: Testing for } \\
\text { Separability in Rural China }\end{array}$ & Audra J. Bowlus and Terry Sicular & January 1999 \\
\hline $\begin{array}{l}\text { No. 218: Competing Strategies of FDI and } \\
\text { Technology Transfer to China: American and } \\
\text { Japanese Firms }\end{array}$ & W. Mark Fruin and Penelope Prime & January 1999 \\
\hline $\begin{array}{l}\text { No. } 217 \text { Published in: Journal of } \\
\text { Comparative Economics, "Returns to } \\
\text { Mobility in the Transition to a Market } \\
\text { Economy" Vol. 27, No. 1, March 1999, pp. 4- }\end{array}$ & Tito Boeri and Christopher J. Flinn & January 1999 \\
\hline $\begin{array}{l}\text { No. } 216 \text { Published in: Journal of } \\
\text { Comparative Economics, "Labor Market } \\
\text { Policies and Unemployment in the Czech } \\
\text { Republic." Vol. 27, No. 1, March 1999, pp. } \\
\text { 33-60. }\end{array}$ & Katherine Terrell and Vit Sorm & November 1998 \\
\hline $\begin{array}{l}\text { No. } 215 \text { Published in: Journal of } \\
\text { Comparative Economics, “Active Labor } \\
\text { Market Policies in Poland: Human Capital } \\
\text { Enhancement, Stigmatization or Benefit } \\
\text { Churning?” Vol. 27, No. 1, March 1999, pp. } \\
\text { 61- }\end{array}$ & $\begin{array}{l}\text { Jochen Kluve, Hartmut Lehmann, and } \\
\text { Christoph M. Schmidt }\end{array}$ & December 1998 \\
\hline $\begin{array}{l}\text { No. } 214 \text { Published in: Journal of } \\
\text { Comparative Economics, "Does the Slovenian } \\
\text { Public Work Program Increase Participants' } \\
\text { Chances to Find a Job?" Vol. 27, No.1, } \\
\text { March 1999, pp. 113- }\end{array}$ & Milan Vodopivec & December 1998 \\
\hline $\begin{array}{l}\text { No. } 213 \text { Published in: Journal of } \\
\text { Comparative Economics, "Effects of Active } \\
\text { Labor Market Programs on the Transition } \\
\text { Rate from Unemployment into Regular Jobs in } \\
\text { the Slovak Republic." Vol. 27, No. 1, March } \\
\text { 1999, pp. 90- }\end{array}$ & Martina Lubyova and Jan C. van Ours & December 1998 \\
\hline $\begin{array}{l}\text { No. 212: The Marketing System in Bulgarian } \\
\text { Livestock Production - The Present State and }\end{array}$ & Yordan Staykov, Team Leader & October 1998 \\
\hline
\end{tabular}

Publications denoted by an asterisk are not available on the Kresge Library webpage (http://www.wdi.bus.umich.edu) 


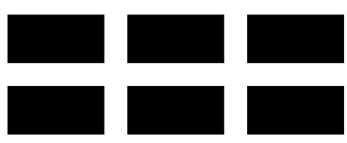

THE WILLIAM DAVIDSON INSTITUTE

AT THE UNIVERSITY OF MICHIGAN BUSINESSSCHOOL

\begin{tabular}{|c|c|c|}
\hline $\begin{array}{l}\text { Evolutionary Processes During the Period of } \\
\text { Economic Transition }\end{array}$ & & \\
\hline $\begin{array}{l}\text { No. 211: Bankruptcy Experience in Hungary } \\
\text { and the Czech Republic }\end{array}$ & Janet Mitchell & October 1998 \\
\hline $\begin{array}{l}\text { No 210: Values, Optimum Stimulation Levels } \\
\text { and Brand Loyalty: New Scales in New } \\
\text { Populations }\end{array}$ & Steven M. Burgess and Mari Harris & September 1998 \\
\hline $\begin{array}{l}\text { No. 209: Inherited Wealth, Corporate Control } \\
\text { and Economic Growth }\end{array}$ & $\begin{array}{l}\text { Randall K. Morck, David A. Stangeland, } \\
\text { and Bernard Yeung }\end{array}$ & September 1998 \\
\hline $\begin{array}{l}\text { No. 208: A Cultural Analysis of Homosocial } \\
\text { Reproduction and Contesting Claims to } \\
\text { Competence in Transitional Firms }\end{array}$ & Michael D. Kennedy & July 1998 \\
\hline $\begin{array}{l}\text { No. 207: From Survival to Success: The } \\
\text { Journey of Corporate Transformation at } \\
\text { Haier. Forthcoming in Teaching the } \\
\text { Dinosaurs to Dance: Organizational Change } \\
\text { in Transition Economies ed. Daniel Denison. } \\
\text { No. 206: Why Do People Work If They Are } \\
\text { Not Paid? An Example from Eastern Europe. } \\
\text { Forthcoming in Teaching the Dinosaurs to } \\
\text { Dance: Organizational Change in Transition } \\
\text { Economies ed. Daniel Denison. }\end{array}$ & $\begin{array}{l}\text { Arthur Yeung and Kenneth DeWoskin } \\
\text { Irina L. Zinovieva }\end{array}$ & $\begin{array}{l}\text { July } 1998 \\
\text { May } 1998\end{array}$ \\
\hline $\begin{array}{l}\text { No. 205: Firm Ownership and Work } \\
\text { Motivation in Bulgaria and Hungary: An } \\
\text { Empirical Study of the Transition in the Mid- } \\
\text { 1990s. Forthcoming in Teaching the } \\
\text { Dinosaurs to Dance: Organizational Change } \\
\text { in Transition Economies ed. Daniel Denison. }\end{array}$ & $\begin{array}{l}\text { Robert A. Roe, Irina L. Zinovieva, } \\
\text { Elizabeth Dienes, and Laurens A. ten Horn }\end{array}$ & May 1998 \\
\hline $\begin{array}{l}\text { No. 204: Human Resource Management in the } \\
\text { Restructuring of Chinese Joint Ventures. } \\
\text { Forthcoming in Teaching the Dinosaurs to } \\
\text { Dance: Organizational Change in Transition } \\
\text { Economies ed. Daniel Denison. }\end{array}$ & Nandani Lynton & April 1998 \\
\hline $\begin{array}{l}\text { No. 203: Emergent Compensation Strategies } \\
\text { in Post-Socialist Poland: Understanding the } \\
\text { Cognitive Underpinnings of Management } \\
\text { Practices in a Transition Economy. } \\
\text { Forthcoming in Teaching the Dinosaurs to } \\
\text { Dance: Organizational Change in Transition } \\
\text { Economies ed. Daniel Denison. }\end{array}$ & Marc Weinstein & March 1998 \\
\hline $\begin{array}{l}\text { No. 202: Corporate Transformation and } \\
\text { Organizational Learning: The People's } \\
\text { Republic of China. Forthcoming in Teaching } \\
\text { the Dinosaurs to Dance: Organizational } \\
\text { Change in Transition Economies ed. Daniel } \\
\text { Denison. }\end{array}$ & Meinolf Dierkes and Zhang Xinhua & March 1998 \\
\hline $\begin{array}{l}\text { No. 201: Foreign Direct Investment as a } \\
\text { Factor of Change: The Case of Slovakia. } \\
\text { Forthcoming in Teaching the Dinosaurs to } \\
\text { Dance: Organizational Change in Transition } \\
\text { Economies ed. Daniel Denison. }\end{array}$ & Sonia Ferencikova & February 1998 \\
\hline No. 200: Radical versus Incremental Change: & Karen L. Newman & February 1998 \\
\hline
\end{tabular}

Publications denoted by an asterisk are not available on the Kresge Library webpage (http://www.wdi.bus.umich.edu) 


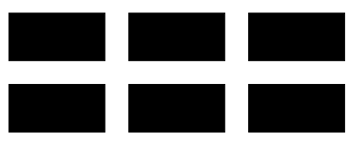

THE WILLIAM DAVIDSON INSTITUTE

AT THE UNIVERSITY OF MICHIGAN BUSINESSSCHOOL

\begin{tabular}{|c|c|c|}
\hline $\begin{array}{l}\text { The Role of Capabilities, Competition, and } \\
\text { Leaders. Forthcoming in Teaching the } \\
\text { Dinosaurs to Dance: Organizational Change } \\
\text { in Transition Economies ed. Daniel Denison. }\end{array}$ & & \\
\hline $\begin{array}{l}\text { No. 199: The Emergence of Market Practices } \\
\text { in China's Economic Transition: Price Setting } \\
\text { Practices in Shanghai's Industrial Firms. } \\
\text { Forthcoming in Teaching the Dinosaurs to } \\
\text { Dance: Organizational Change in Transition } \\
\text { Economies ed. Daniel Denison. }\end{array}$ & Douglas Guthrie & February 1998 \\
\hline $\begin{array}{l}\text { No. 198: The Application of Change } \\
\text { Management Methods at Business } \\
\text { Organizations Operating in Hungary: } \\
\text { Challenges in the Business and Cultural } \\
\text { Environment and First Practical Experiences. } \\
\text { Forthcoming in Teaching the Dinosaurs to } \\
\text { Dance: Organizational Change in Transition } \\
\text { Economies ed. Daniel Denison. }\end{array}$ & Dr. János Fehér & January 1998 \\
\hline $\begin{array}{l}\text { No. 197: Organizational Changes in Russian } \\
\text { Industrial Enterprises: Mutation of Decision- } \\
\text { Making Structures and Transformations of } \\
\text { Ownership. Forthcoming in Teaching the } \\
\text { Dinosaurs to Dance: Organizational Change } \\
\text { in Transition Economies ed. Daniel Denison. }\end{array}$ & Igor B. Gurkov & January 1998 \\
\hline $\begin{array}{l}\text { No. 196: Understanding and Managing } \\
\text { Challenges to the Romanian Companies } \\
\text { during Transition. Forthcoming in Teaching } \\
\text { the Dinosaurs to Dance: Organizational } \\
\text { Change in Transition Economies ed. Daniel } \\
\text { Denison. }\end{array}$ & Dan Candea and Rodica M. Candea & January 1998 \\
\hline $\begin{array}{l}\text { No. 195: Insider Lending and Economic } \\
\text { Transition: The Structure, Function, and } \\
\text { Performance Impact of Finance Companies in } \\
\text { Chinese Business Groups. Forthcoming in } \\
\text { Teaching the Dinosaurs to Dance: } \\
\text { Organizational Change in Transition } \\
\text { Economies ed. Daniel Denison. }\end{array}$ & Lisa A. Keister & December 1997 \\
\hline $\begin{array}{l}\text { No. 194: Japanese Investment in Transitional } \\
\text { Economies: Characteristics and Performance. } \\
\text { Forthcoming in Teaching the Dinosaurs to } \\
\text { Dance: Organizational Change in Transition } \\
\text { Economies ed. Daniel Denison. }\end{array}$ & Paul W. Beamish and Andrew Delios & November 1997 \\
\hline $\begin{array}{l}\text { No. 193: Building Successful Companies in } \\
\text { Transition Economies. Forthcoming in } \\
\text { Teaching the Dinosaurs to Dance: } \\
\text { Organizational Change in Transition } \\
\text { Economies ed. Daniel Denison. }\end{array}$ & Dr. Ivan Perlaki & January 1998 \\
\hline $\begin{array}{l}\text { No. 192: Russian Communitariansim: An } \\
\text { Invisible Fist in the Transformation Process of } \\
\text { Russia. Forthcoming in Teaching the } \\
\text { Dinosaurs to Dance: Organizational Change } \\
\text { in Transition Economies ed. Daniel Denison. }\end{array}$ & Charalambos Vlachoutsicos & July 1998 \\
\hline
\end{tabular}

Publications denoted by an asterisk are not available on the Kresge Library webpage (http://www.wdi.bus.umich.edu) 


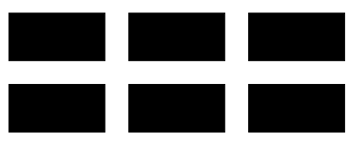

THE WILLIAM DAVIDSON INSTITUTE

AT THE UNIVERSITY OF MICHIGAN BUSINESSSCHOOL

\begin{tabular}{|c|c|c|}
\hline No. 191: Teaching the Dinosaurs to Dance & Michal Cakrt & September 1997 \\
\hline $\begin{array}{l}\text { No. 190: Strategic Restructuring: Making } \\
\text { Capitalism in Post-Communist Eastern } \\
\text { Europe. Forthcoming in Teaching the } \\
\text { Dinosaurs to Dance: Organizational Change } \\
\text { in Transition Economies ed. Daniel Denison. }\end{array}$ & Lawrence P. King & September 1997 \\
\hline $\begin{array}{l}\text { No. 189: Published in: Regional Science and } \\
\text { Urban Economics, "Russia's Internal } \\
\text { Border", 29 (5), September 1999. }\end{array}$ & Daniel Berkowitz and David N. DeJong & July 1998 \\
\hline $\begin{array}{l}\text { No. 187: Corporate Structure and } \\
\text { Performance in Hungary }\end{array}$ & László Halpern and Gábor Kórsöi & July 1998 \\
\hline $\begin{array}{l}\text { No. 186: Performance of Czech Companies by } \\
\text { Ownership Structure }\end{array}$ & Andrew Weiss and Georgiy Nikitin & June 1998 \\
\hline $\begin{array}{l}\text { No. 185: Firm Performance in Bulgaria and } \\
\text { Estonia: The effects of competitive pressure, } \\
\text { financial pressure and disorganisation }\end{array}$ & Jozef Konings & July 1998 \\
\hline $\begin{array}{l}\text { No. 184: Investment and Wages during the } \\
\text { Transition: Evidence from Slovene Firms }\end{array}$ & Janez Prasnikar and Jan Svejnar & July 1998 \\
\hline $\begin{array}{l}\text { No. 183: Investment Portfolio under Soft } \\
\text { Budget: Implications for Growth, Volatility } \\
\text { and Savings }\end{array}$ & Chongen Bai and Yijiang Wang & \\
\hline $\begin{array}{l}\text { No. 181: Delegation and Delay in Bank } \\
\text { Privatization }\end{array}$ & Loránd Ambrus-Lakatos and Ulrich Hege & July 1998 \\
\hline $\begin{array}{l}\text { No. 180: Financing Mechanisms and } R \& D \\
\text { Investment }\end{array}$ & Haizhou Huang and Chenggang $X u$ & July 1998 \\
\hline $\begin{array}{l}\text { No. 179: Organizational Culture and } \\
\text { Effectiveness: The Case of Foreign Firms in } \\
\text { Russia }\end{array}$ & Carl F. Fey and Daniel R. Denison & January 1999 \\
\hline $\begin{array}{l}\text { No. 178: Output and Unemployment } \\
\text { Dynamics in Transition }\end{array}$ & Vivek H. Dehejia and Douglas W. Dwyer & January 1998 \\
\hline $\begin{array}{l}\text { No. 177: Published in: Economics of } \\
\text { Transition,, "Bureaucracies in the Russian } \\
\text { Voucher Privatization" Vol. 8, No. 1, 2000, } \\
\text { pp. } 37-57 \text {. }\end{array}$ & Guido Friebel & June 1998 \\
\hline $\begin{array}{l}\text { No. 176: Chronic Moderate Inflation in } \\
\text { Transition: The Tale of Hungary }\end{array}$ & János Vincze & June 1998 \\
\hline $\begin{array}{l}\text { No. 175: Privatisation and Market Structure } \\
\text { in a Transition Economy }\end{array}$ & John Bennett and James Maw & June 1998 \\
\hline $\begin{array}{l}\text { No. 174: Ownership and Managerial } \\
\text { Competition: Employee, Customer, or Outside } \\
\text { Ownership }\end{array}$ & Patrick Bolton and Chenggang $X u$ & June 1998 \\
\hline $\begin{array}{l}\text { No. 173: Intragovernment Procurement of } \\
\text { Local Public Good: A Theory of } \\
\text { Decentralization in Nondemocratic } \\
\text { Government }\end{array}$ & Chong-en Bai, Yu Pan and Yijiang Wang & June 1998 \\
\hline $\begin{array}{l}\text { No. 172: Political Instability and Growth in } \\
\text { Proprietary Economies }\end{array}$ & Jody Overland and Michael Spagat & August 1998 \\
\hline $\begin{array}{l}\text { No. 171: Published in Post-Communist } \\
\text { Economies, "Framework Issues in the } \\
\text { Privatization Strategies of the Czech Republic, } \\
\text { Hungary, and Poland" Vol. 11, no. 1 March } \\
\text { 1999. }\end{array}$ & Morris Bornstein & June 1998 \\
\hline
\end{tabular}

Publications denoted by an asterisk are not available on the Kresge Library webpage (http://www.wdi.bus.umich.edu) 


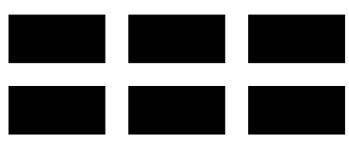

THE WILliam DAVIDSON INSTITUTE

AT THE UNIVERSITY OF MICHIGAN BUSINESSSCHOOL

\begin{tabular}{|c|c|c|}
\hline $\begin{array}{l}\text { No. 170: Published in: European Journal of } \\
\text { Political Economy "Privatization, Ownership } \\
\text { Structure and Transparency: How to Measure } \\
\text { a Real Involvement of the State" 15(4), } \\
\text { November 1999, pp. 605-18. }\end{array}$ & Frantisek Turnovec & May 1998 \\
\hline $\begin{array}{l}\text { No. } 169 \text { Published in: American Economic } \\
\text { Review, "Unemployment and the Social Safety } \\
\text { Net during Transitions to a Market Economy: } \\
\text { Evidence from Czech and Slovak Men." Vol. } \\
\text { 88, No. 5, Dec. 1998, pp. 1117-1142. }\end{array}$ & $\begin{array}{l}\text { John C. Ham, Jan Svejnar, and Katherine } \\
\text { Terrell }\end{array}$ & December 1998 \\
\hline $\begin{array}{l}\text { No. 167: Voucher Privatization with } \\
\text { Investment Funds: An Institutional Analysis }\end{array}$ & David Ellerman & March 1998 \\
\hline $\begin{array}{l}\text { No. 166: Published in: Marketing Issues in } \\
\text { Transitional Economies, "Value Priorities } \\
\text { and Consumer Behavior in a Transitional } \\
\text { Economy: The Case of South Africa" ed. } \\
\text { Rajeev Batra. }\end{array}$ & $\begin{array}{l}\text { Steven M. Burgess and Jan-Benedict E.M. } \\
\text { Steenkamp }\end{array}$ & August 1998 \\
\hline $\begin{array}{l}\text { No. 164: Finance and Investment in } \\
\text { Transition: Czech Enterprises, 1993-1994 }\end{array}$ & Ronald Anderson and Chantal Kegels & September 1997 \\
\hline $\begin{array}{l}\text { No. 163: European Union Trade and } \\
\text { Investment Flows U-Shaping Industrial } \\
\text { Output in Central and Eastern Europe: } \\
\text { Theory and Evidence }\end{array}$ & Alexander Repkine and Patrick P. Walsh & April 1998 \\
\hline $\begin{array}{l}\text { No. 162: Skill Acquisition and Private Firm } \\
\text { Creation in Transition Economies }\end{array}$ & Zuzana Brixiova and Wenli Li & October 1999 \\
\hline No. 161: Corruption in Transition & Susanto Basu and David D. Li & May 1998 \\
\hline $\begin{array}{l}\text { No. 160a: Tenures that Shook the World: } \\
\text { Worker Turnover in Russia, Poland and } \\
\text { Britain }\end{array}$ & $\begin{array}{l}\text { Hartmut Lehmann and Jonathan } \\
\text { Wadsworth }\end{array}$ & November 1999 \\
\hline $\begin{array}{l}\text { No. 160: Tenures that Shook the World: } \\
\text { Worker Turnover in the Russian Federation } \\
\text { and Poland }\end{array}$ & $\begin{array}{l}\text { Hartmut Lehmann and Jonathan } \\
\text { Wadsworth }\end{array}$ & June 1998 \\
\hline $\begin{array}{l}\text { No. 159: Does Market Structure Matter? New } \\
\text { Evidence from Russia }\end{array}$ & Annette N. Brown and J. David Brown & June 1998 \\
\hline $\begin{array}{l}\text { No. 158: Structural Adjustment and Regional } \\
\text { Long Term Unemployment in Poland }\end{array}$ & Hartmut Lehmann and Patrick P. Walsh & June 1997 \\
\hline $\begin{array}{l}\text { No. 157: Baby Boom or Bust? Changing } \\
\text { Fertility in Post-Communist Czech Republic } \\
\text { and Slovakia }\end{array}$ & Robert S. Chase & April 1998 \\
\hline $\begin{array}{l}\text { No. } 156 \text { Published in: Leadership and } \\
\text { Organization Development Journal, } \\
\text { "Leading Radical Change in Transition } \\
\text { Economies." Vol. 19, No. 6, 1998, pp. 309- } \\
324 .\end{array}$ & Karen L. Newman & June 1998 \\
\hline $\begin{array}{l}\text { No. } 155 \text { Published in: Oxford Review of } \\
\text { Economic Policy, "From Theory into } \\
\text { Practice? Restructuring and Dynamism in } \\
\text { Transition Economies." Vol. 13, No. 2, } \\
\text { Summer 1997, pp. 77-105. }\end{array}$ & Wendy Carlin and Michael Landesmann & June 1997 \\
\hline $\begin{array}{l}\text { No. 154: The Model and the Reality: } \\
\text { Assessment of Vietnamese SOE Reform- } \\
\text { Implementation at the Firm Level }\end{array}$ & $\begin{array}{l}\text { Edmund Malesky, Vu Thanh Hung, Vu Thi } \\
\text { Dieu Anh, and Nancy K. Napier }\end{array}$ & July 1998 \\
\hline
\end{tabular}

Publications denoted by an asterisk are not available on the Kresge Library webpage (http://www.wdi.bus.umich.edu) 


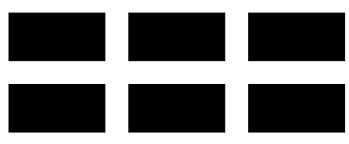

THE WILLIAM DAVIDSON INSTITUTE

AT THE UNIVERSITY OF MICHIGAN BUSINESSSCHOOL

\begin{tabular}{|c|c|c|}
\hline $\begin{array}{l}\text { No. } 153 \text { Published in: Journal of } \\
\text { Comparative Economics, "Causes of the Soft } \\
\text { Budget Constraint: Evidence on Three } \\
\text { Explanations." Vol. 26, No. 1, March 1998, } \\
\text { pp. 104-116. }\end{array}$ & David D. Li and Minsong Liang & March 1998 \\
\hline $\begin{array}{l}\text { No. } 152 \text { Published in: Comparative Economic } \\
\text { Studies, "Enterprise Restructuring in Russia's } \\
\text { Transition Economy: Formal and Informal } \\
\text { Mechanisms." Vol. 40, No. 2, Summer 1998, } \\
\text { pp. 5-52. }\end{array}$ & Susan J. Linz and Gary Krueger & April 1998 \\
\hline $\begin{array}{l}\text { No. 151: Labor Productivity in Transition: A } \\
\text { Regional Analysis of Russian Industry }\end{array}$ & Susan J. Linz & May 1998 \\
\hline $\begin{array}{l}\text { No. 150: Tax Avoidance and the Allocation of } \\
\text { Credit. Forthcoming in Financial Systems in } \\
\text { Transition: The Design of Financial Systems } \\
\text { in Central Europe eds. Anna Meyendorff and } \\
\text { Anjan Thakor. }\end{array}$ & Anna Meyendorff & June 1998 \\
\hline $\begin{array}{l}\text { No. 149: Commitment, Versatility and } \\
\text { Balance: Determinants of Work Time } \\
\text { Standards and Norms in a Multi-Country } \\
\text { Study of Software Engineers }\end{array}$ & Leslie Perlow and Ron Fortgang & April 1998 \\
\hline $\begin{array}{l}\text { No. 148: Changes in Poland's Transfer } \\
\text { Payments in the 1990s: the Fate of } \\
\text { Pensioners }\end{array}$ & Bozena Leven & June 1998 \\
\hline $\begin{array}{l}\text { No. 147: Environmental Protection and } \\
\text { Economic Development: The Case of the } \\
\text { Huaihe River Basin Cleanup Plan }\end{array}$ & $\begin{array}{l}\text { Robert Letovsky, Reze Ramazani, and } \\
\text { Debra Murphy }\end{array}$ & June 1998 \\
\hline $\begin{array}{l}\text { No. 146: Chief Executive Compensation } \\
\text { During Early Transition: Further Evidence } \\
\text { from Bulgaria }\end{array}$ & $\begin{array}{l}\text { Derek C. Jones, Takao Kato, and Jeffrey } \\
\text { Miller }\end{array}$ & June 1998 \\
\hline $\begin{array}{l}\text { No. } 145 \text { Published in: Economics of } \\
\text { Transition, "Women's Unemployment During } \\
\text { the Transition: Evidence from Czech and } \\
\text { Slovak Micro Data," Vol. 7, No. 1, May 1999, } \\
\text { pp. 47-78. }\end{array}$ & $\begin{array}{l}\text { John Ham, Jan Svejnar, and Katherine } \\
\text { Terrell }\end{array}$ & May 1998 \\
\hline No. 144: Investment and Wages in Slovenia & Janez Prasnikar & May 1998 \\
\hline $\begin{array}{l}\text { No. } 143 \text { Published in: Review of Financial } \\
\text { Studies, “Optimal Bankruptcy Laws Across } \\
\text { Different Economic Systems," 12(2), Summer } \\
\text { 1999, pgs. 347-77. }\end{array}$ & Elazar Berkovitch and Ronen Israel & March 1998 \\
\hline $\begin{array}{l}\text { No. 142: Industrial Policy and Poverty in } \\
\text { Transition Economies: Two Steps Forward or } \\
\text { One Step Back? }\end{array}$ & Susan J. Linz & March 1998 \\
\hline $\begin{array}{l}\text { No. 141: Collective Ownership and } \\
\text { Privatization of China's Village Enterprises }\end{array}$ & Suwen Pan and Albert Park & April 1998 \\
\hline $\begin{array}{l}\text { No. 140: A Comparative Look at Labor } \\
\text { Mobility in the Czech Republic: Where have } \\
\text { all the Workers Gone? }\end{array}$ & Vit Sorm and Katherine Terrell & April 1999 \\
\hline $\begin{array}{l}\text { No. 139: The Failure of the Government-Led } \\
\text { Program of Corporate Reorganization in } \\
\text { Romania }\end{array}$ & Simeon Djankov and Kosali Ilayperuma & September 1997 \\
\hline No. 138: Ownership and Employment in & Susan J. Linz. & March 1998 \\
\hline
\end{tabular}

Publications denoted by an asterisk are not available on the Kresge Library webpage (http://www.wdi.bus.umich.edu) 


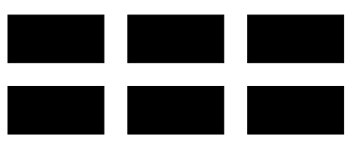

THE WILLIAM DAVIDSON INSTITUTE

AT THE UNIVERSITY OF MICHIGAN BUSINESSSCHOOL

\begin{tabular}{|c|c|c|}
\hline \multirow[b]{2}{*}{\begin{tabular}{|l|} 
Russian Industry: 1992-1995 \\
No. 137 Published in: Journal of Political \\
Economy, "Reform Without Losers: An \\
Interpretation of China's Dual-Track \\
Approach to Transition," Feb. 2000; Vol. 108, \\
Iss.1; pg. 120
\end{tabular}} & & \\
\hline & $\begin{array}{l}\text { Lawrence J. Lau, Yingyi Qian, and Gerard } \\
\text { Roland }\end{array}$ & November 1997 \\
\hline $\begin{array}{l}\text { No. } 136 \text { Published in: European Economic } \\
\text { Review, "The Political Economy of Mass } \\
\text { Privatization and the Risk of Expropriation," } \\
44(2), \text { February 2000, pgs. } 393-421\end{array}$ & Klaus M. Schmidt & March 1998 \\
\hline $\begin{array}{l}\text { No. 135: Radical Organizational Change: The } \\
\text { Role of Starting Conditions, Competition, and } \\
\text { Leaders }\end{array}$ & Karen L. Newman & January 1998 \\
\hline $\begin{array}{l}\text { No. 134: To Restructure or Not to } \\
\text { Restructure: Informal Activities and } \\
\text { Enterprise Behavior in Transition }\end{array}$ & Clifford Gaddy and Barry W. Ickes & May 1998 \\
\hline $\begin{array}{l}\text { No. 133: Management 101: Behavior of Firms } \\
\text { in Transition Economies }\end{array}$ & Josef C. Brada & March 1998 \\
\hline $\begin{array}{l}\text { No. } 132 \text { Published in: Quarterly Journal of } \\
\text { Economics, "Interfirm Relationships and } \\
\text { Informal Credit in Vietnam," 114(4), Nov. } \\
\text { 1999, pgs. 1285-1320 }\end{array}$ & John McMillan and Christopher Woodruff & February 1998 \\
\hline $\begin{array}{l}\text { No. 131 Published in: Comparative Economic } \\
\text { Studies, "Will Restructuring Hungarian } \\
\text { Companies Innovate? An Investigation Based } \\
\text { on Joseph Berliner's Analysis of Innovation in } \\
\text { Soviet Industry.” Vol. 40, No. 2, Summer } \\
\text { 1998, pp. 53-74. }\end{array}$ & John B. Bonin and Istvan Abel & March 1998 \\
\hline $\begin{array}{l}\text { No. 130: Published in The American } \\
\text { Economic Review, "Changing Incentives of } \\
\text { the Chinese Bureaucracy." May, 1998. }\end{array}$ & David D. Li & January 1998 \\
\hline $\begin{array}{l}\text { No. 129: Restructuring Investment in } \\
\text { Transition: A Model of the Enterprise } \\
\text { Decision }\end{array}$ & Richard E. Ericson & January 1998 \\
\hline $\begin{array}{l}\text { No. } 128 \text { Published in: Comparative Economic } \\
\text { Studies, “Job Rights in Russian Firms: } \\
\text { Endangered or Extinct Institutions?" Vol. 40, } \\
\text { No. 4, Winter 1998, pp. 1-32. }\end{array}$ & Susan J. Linz & January 1998 \\
\hline $\begin{array}{l}\text { No. 127: Accounting for Growth in Post- } \\
\text { Soviet Russia }\end{array}$ & Daniel Berkowitz and David N. DeJong & January 1998 \\
\hline $\begin{array}{l}\text { No. } 126 \text { Published in: Economics of } \\
\text { Transition, "From Federalism, Chinese Style, } \\
\text { to Privatization Chinese Style," 7(1), 1999, } \\
\text { pgs. 103-31 }\end{array}$ & $\begin{array}{l}\text { Yuanzheng Cao, Yingyi Qian, and Barry R. } \\
\text { Weingast }\end{array}$ & December 1997 \\
\hline $\begin{array}{l}\text { No. 125: Market Discipline in Conglomerate } \\
\text { Banks: Is an Internal Allocation of Cost of } \\
\text { Capital Necessary as Incentive Device? } \\
\text { Forthcoming in Financial Systems in } \\
\text { Transition: The Design of Financial Systems } \\
\text { in Central Europe eds. Anna Meyendorff and } \\
\text { Anjan Thakor. }\end{array}$ & Arnoud W. A. Boot and Anjolein Schmeits & November 1997 \\
\hline No. 124: Financial Discipline in the & Shumei Gao and Mark E. Schaffer & February 1998 \\
\hline
\end{tabular}

Publications denoted by an asterisk are not available on the Kresge Library webpage (http://www.wdi.bus.umich.edu) 


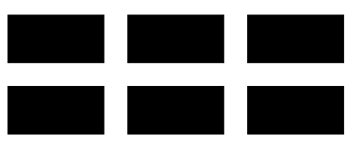

THE WILLIAM DAVIDSON INSTITUTE

AT THE UNIVERSITY OF MICHIGAN BUSINESSSCHOOL

\begin{tabular}{|c|c|c|}
\hline $\begin{array}{l}\text { Enterprise Sector in Transition Countries: } \\
\text { How Does China Compare? }\end{array}$ & & \\
\hline $\begin{array}{l}\text { No. 123: Considerations of an Emerging } \\
\text { Marketplace: Managers' Perceptions in the } \\
\text { Southern African Economic Community }\end{array}$ & Brent Chrite and David Hudson & February 1998 \\
\hline $\begin{array}{l}\text { No. 122: A Model of the Informal Economy in } \\
\text { Transition Economies }\end{array}$ & $\begin{array}{l}\text { Simon Commander and Andrei } \\
\text { Tolstopiatenko }\end{array}$ & November 1997 \\
\hline $\begin{array}{l}\text { No. 121: Local Labour Market Dynamics in } \\
\text { the Czech and Slovak Republics }\end{array}$ & Peter Huber and Andreas Worgotter & November 1997 \\
\hline $\begin{array}{l}\text { No. 121: Local Labour Market Dynamics in } \\
\text { the Czech and Slovak Republics }\end{array}$ & Peter Huber and Andreas Worgotter & November 1997 \\
\hline $\begin{array}{l}\text { No. 119: Institutional Upheaval and Company } \\
\text { Transformation in Emerging Market } \\
\text { Economies }\end{array}$ & Karen L. Newman & March 1998 \\
\hline $\begin{array}{l}\text { No. 118: Industrial Decline and Labor } \\
\text { Reallocation in Romania }\end{array}$ & John S. Earle & October 1997 \\
\hline $\begin{array}{l}\text { No. 117: Notes for an Essay on the Soft } \\
\text { Budget Constraint }\end{array}$ & Lorand Ambrus-Lakatos & January 1997 \\
\hline $\begin{array}{l}\text { No. 116: Labor Demand During Transition in } \\
\text { Hungary }\end{array}$ & Gabor Korosi & October 1997 \\
\hline $\begin{array}{l}\text { No. 115: Enterprise Performance and } \\
\text { Managers' Profiles }\end{array}$ & Simeon Djankov and Stijn Claessens & December 1997 \\
\hline $\begin{array}{l}\text { No. } 114 b \text { Employment and Wages in } \\
\text { Enterprises under Communism and in } \\
\text { Transition: Evidence From Central Europe } \\
\text { and Russia }\end{array}$ & Swati Basu, Saul Estrin, and Jan Svejnar & April 2000 \\
\hline $\begin{array}{l}\text { No. 114: Employment and Wage Behavior of } \\
\text { Enterprises in Transitional Economies }\end{array}$ & Swati Basu, Saul Estrin, and Jan Svejnar & October 1997 \\
\hline $\begin{array}{l}\text { No. 113: Preliminary Evidence on Active } \\
\text { Labor Programs' Impact in Hungary and } \\
\text { Poland }\end{array}$ & Christopher J. O’Leary & October 1997 \\
\hline $\begin{array}{l}\text { No. 111: Unemployment Benefits and } \\
\text { Incentives in Hungary: New Evidence }\end{array}$ & Joachim Wolff & October 1997 \\
\hline $\begin{array}{l}\text { No. 110: Published in: Empirical Economics, } \\
\text { "Long-Term Unemployment, Unemployment } \\
\text { Benefits and Social Assistance: The Polish } \\
\text { Experience" Empirical-Economics; 23(1-2), } \\
\text { 1998, pages 55-85. }\end{array}$ & Marek Gora and Christoph M. Schmidt & April 1997 \\
\hline $\begin{array}{l}\text { No. } 109 \text { Published in: Industrial and Labor } \\
\text { Relations Review, "Markets for Communist } \\
\text { Human Capital: Returns to Education and } \\
\text { Experience in Post-Communist Czech } \\
\text { Republic and Slovakia." Vol. 51, No. 3, April } \\
\text { 1998, pp. 401-423. }\end{array}$ & Robert S. Chase & October 1997 \\
\hline $\begin{array}{l}\text { No. 107: The Worker-Firm Matching in the } \\
\text { Transition: (Why) Are the Czechs More } \\
\text { Successful Than Others? }\end{array}$ & $\begin{array}{l}\text { Daniel Münich, Jan Svejnar, and } \\
\text { Katherine Terrell }\end{array}$ & October 1997 \\
\hline $\begin{array}{l}\text { No. } 106 \text { Published in: Journal of } \\
\text { Comparative Economics, "Job Creation, Job } \\
\text { Destruction and Growth of Newly Established, } \\
\text { Privatized and State-Owned Enterprises in } \\
\text { Transition Economies: Survey Evidence from }\end{array}$ & Valentijn Bilsen and Jozef Konings & September 1998 \\
\hline
\end{tabular}

Publications denoted by an asterisk are not available on the Kresge Library webpage (http://www.wdi.bus.umich.edu) 


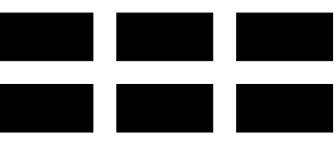

THE WILLIAM DAVIDSON INSTITUTE

AT THE UNIVERSITY OF MICHIGAN BUSINESSSCHOOL

\begin{tabular}{|c|c|c|}
\hline $\begin{array}{l}\text { Bulgaria, Hungary, and Romania," Vol. 26, } \\
\text { No.3, September 1998, pp. 429-445. }\end{array}$ & & \\
\hline $\begin{array}{l}\text { No. 105: Getting Behind the East-West } \\
\text { [German] Wage Differential: Theory and } \\
\text { Evidence }\end{array}$ & Michael Burda and Christoph Schmidt & May 1997 \\
\hline $\begin{array}{l}\text { No. 104: The Birth of the "Wage Curve" in } \\
\text { Hungary, 1989-95 }\end{array}$ & Gabor Kertesi and Janos Kollo & October 1997 \\
\hline $\begin{array}{l}\text { No. 103: Published in: Journal of } \\
\text { Comparative Economics, "Grime and } \\
\text { Punishment: Job Insecurity and Wage Arrears } \\
\text { in the Russian Federation" 27, 595-617 } \\
\text { (1999). }\end{array}$ & $\begin{array}{l}\text { Hartmut Lehmann, Jonathan Wadsworth, } \\
\text { and Alessandro Acquisti }\end{array}$ & October 1997 \\
\hline No. 102: Social Networks in Transition & $\begin{array}{l}\text { Lorena Barberia, Simon Johnson, and } \\
\text { Daniel Kaufmann }\end{array}$ & October 1997 \\
\hline $\begin{array}{l}\text { No. 101: Depreciation and Russian Corporate } \\
\text { Finance: A Pragmatic Approach to Surviving } \\
\text { the Transition }\end{array}$ & Susan J. Linz & November 1997 \\
\hline No. 100: Romanian Financial System Reform & Anna Meyendorff and Anjan $V$. Thakor & November 1997 \\
\hline $\begin{array}{l}\text { No. 99: Proceedings of the Conference on } \\
\text { Strategic Alliances in Transitional Economies, } \\
\text { held May 20, } 1997 \text { at the Davidson Institute }\end{array}$ & Edited by Cynthia Koch & May 1997 \\
\hline $\begin{array}{l}\text { No. 98: Institutions, Strain and the } \\
\text { Underground Economy }\end{array}$ & Daniel Daianu and Lucian Albu & November 1997 \\
\hline $\begin{array}{l}\text { No. 97: Structure and Strain in Explaining } \\
\text { Inter-Enterprise Arrears } \\
\text { No. 96: Resource Misallocation and Strain: } \\
\text { Explaining Shocks in Post-Command } \\
\text { Economies }\end{array}$ & $\begin{array}{l}\text { Daniel Daianu } \\
\text { Daniel Daianu }\end{array}$ & $\begin{array}{l}\text { November } 1997 \\
\text { November } 1997\end{array}$ \\
\hline $\begin{array}{l}\text { No. 95: Published in: Finance-a-Uver, } \\
\text { “Czech Money Market: Emerging Links } \\
\text { Among Interest Rates." 48(2) } 1998 \text { pp. 99- } \\
\text { 109. }\end{array}$ & Jan Hanousek and Evzen Kocenda & November 1997 \\
\hline $\begin{array}{l}\text { No. 94: Pre-Reform Industry and the } \\
\text { State Monopsony in China }\end{array}$ & Xiao-Yuan Dong and Louis Putterman & October 1997 \\
\hline $\begin{array}{l}\text { No. 93: China's State-Owned Enterprises } \\
\text { In the First Reform Decade: } \\
\text { An Analysis of a Declining Monopsony }\end{array}$ & Xiao-Yuan Dong and Louis Putterman & October 1997 \\
\hline $\begin{array}{l}\text { No. 92: Expatriate Management in the Czech } \\
\text { Republic }\end{array}$ & Richard B. Peterson & September 1997 \\
\hline $\begin{array}{l}\text { No. 91: China and the Idea of Economic } \\
\text { Reform }\end{array}$ & Thomas G. Rawski & April 1997 \\
\hline $\begin{array}{l}\text { No. } 90 \text { Published in: China Economic } \\
\text { Review, “China's State Enterprise Reform: An } \\
\text { Overseas Perspective." Vol. 8, Spring 1997, } \\
\text { pp. 89-98. }\end{array}$ & Thomas G. Rawski & July 1997 \\
\hline $\begin{array}{l}\text { No. 89: The Economic Determinants of } \\
\text { Internal Migration Flows in Russia During } \\
\text { Transition }\end{array}$ & Annette N. Brown & July 1997 \\
\hline $\begin{array}{l}\text { No. 88: Gender Wage Gaps in China's Labor } \\
\text { Market: Size, Structure, Trends }\end{array}$ & $\begin{array}{l}\text { Margaret Maurer-Fazio, Thomas G. } \\
\text { Rawski, and Wei Zhang }\end{array}$ & July 1997 \\
\hline $\begin{array}{l}\text { No. 87: Privatisation in Central and Eastern } \\
\text { Europe }\end{array}$ & Saul Estrin & June 1997 \\
\hline
\end{tabular}

Publications denoted by an asterisk are not available on the Kresge Library webpage (http://www.wdi.bus.umich.edu) 


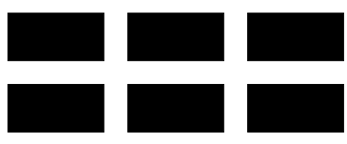

THE WILLIAM DAVIDSON INSTITUTE AT THE UNIVERSITY OF MICHIGAN BUSINESSSCHOOL

\begin{tabular}{|c|c|c|}
\hline $\begin{array}{l}\text { No. 86: Published in : Economics of } \\
\text { Transition, "The Effect of Privatization on } \\
\text { Wealth Distribution in Russia." v. 7, no. 2, } \\
\text { 1999, pp. 449-65 }\end{array}$ & Michael Alexeev & February 1998 \\
\hline $\begin{array}{l}\text { No. 85: Was Privatization in Eastern Germany } \\
\text { a Special Case? Some Lessons from the } \\
\text { Treuhand }\end{array}$ & Uwe Siegmund & September 1997 \\
\hline No. 84: Start-ups and Transition & Daniel M. Berkowitz and David J. Cooper & September 1997 \\
\hline $\begin{array}{l}\text { No. 83: Which Enterprises (Believe They) } \\
\text { Have Soft Budgets after Mass Privatization? } \\
\text { Evidence from Mongolia }\end{array}$ & $\begin{array}{l}\text { James Anderson, Georges Korsun, and } \\
\text { Peter Murrell }\end{array}$ & October 1997 \\
\hline $\begin{array}{l}\text { No. 82: Published in: European Economic } \\
\text { Review, "Unemployment Dynamics and the } \\
\text { Restructuring of the Slovak Unemployment } \\
\text { Benefit System." April, 1997. }\end{array}$ & Martina Lubyova and Jan C. van Ours & June 1997 \\
\hline $\begin{array}{l}\text { No. 81: Determinants of Unemployment } \\
\text { Duration in Russia }\end{array}$ & Mark C. Foley & August 1997 \\
\hline $\begin{array}{l}\text { No. 80: The Many Faces of Information } \\
\text { Disclosure }\end{array}$ & Arnoud W.A. Boot and Anjan V. Thakor & October 1997 \\
\hline $\begin{array}{l}\text { No. 79: Published in: Journal of Finance, } \\
\text { "Foreign Speculators and Emerging Equity } \\
\text { Markets."v.22, iss. 2, 2000, pp. 565-613 }\end{array}$ & Geert Bekaert and Campbell R. Harvey & August 1997 \\
\hline $\begin{array}{l}\text { No. 78: The Relationship Between Economic } \\
\text { Factors and Equity Markets in Central Europe }\end{array}$ & Jan Hanousek and Randall K. Filer & June 1997 \\
\hline $\begin{array}{l}\text { No. } 77 \text { Published in: Economics of } \\
\text { Transition, "A Gini Decomposition Analysis } \\
\text { of Inequality in the Czech and Slovak } \\
\text { Republics During the Transition," Vol. 6, } \\
\text { No.1, May 1998, pp. 23-46. }\end{array}$ & Thesia I. Garner and Katherine Terrell & May 1998 \\
\hline $\begin{array}{l}\text { No. 76: China's Emerging Market for } \\
\text { Property Rights: Theoretical and Empirical } \\
\text { Perspectives }\end{array}$ & Gary H. Jefferson and Thomas G. Rawski & June 1997 \\
\hline $\begin{array}{l}\text { No. } 75 b: \text { Test of Permanent Income } \\
\text { Hypothesis on Czech Voucher Privatization }\end{array}$ & Jan Hanousek and Zdenek Tima & October 1997 \\
\hline $\begin{array}{l}\text { No. 74: Determinants of Performance of } \\
\text { Manufacturing Firms in Seven European } \\
\text { Transition Economies }\end{array}$ & $\begin{array}{l}\text { Stijn Claessens, Simeon Djankov, and } \\
\text { Gerhard Pohl }\end{array}$ & February 1997 \\
\hline $\begin{array}{l}\text { No. } 73 \text { Published in: Economics of } \\
\text { Transition, “The Restructuring of Large } \\
\text { Firms in Slovak Republic." Vol. 6, No. 1, May } \\
\text { 1998, pp. 67-85 }\end{array}$ & Simeon Djankov and Gerhard Pohl & May 1998 \\
\hline $\begin{array}{l}\text { No. 72: Law, Relationships, and Private } \\
\text { Enforcement: Transactional Strategies of } \\
\text { Russian Enterprises }\end{array}$ & $\begin{array}{l}\text { Kathryn Hendley, Peter Murrell, and } \\
\text { Randi Ryterman }\end{array}$ & November 1998 \\
\hline $\begin{array}{l}\text { No. 71: Giving Credit Where Credit Is Due: } \\
\text { The Changing Role of Rural Financial } \\
\text { Institutions in China }\end{array}$ & Albert Park, Loren Brandt, and John Giles & March 1997 \\
\hline $\begin{array}{l}\text { No. 70: Privatization Versus Competition: } \\
\text { Changing Enterprise Behavior in Russia }\end{array}$ & John S. Earle and Saul Estrin & Spring 1997 \\
\hline $\begin{array}{l}\text { No. 69: Russian Managers under Storm: } \\
\text { Explicit Reality and Implicit Leadership } \\
\text { Theories (A Pilot Exploration) }\end{array}$ & Igor Gurkov & October 1998 \\
\hline
\end{tabular}

Publications denoted by an asterisk are not available on the Kresge Library webpage (http://www.wdi.bus.umich.edu) 


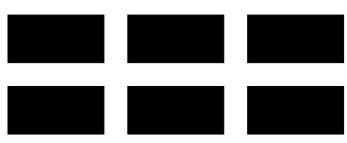

THE WILLIAM DAVIDSON INSTITUTE

AT THE UNIVERSITY OF MICHIGAN BUSINESSSCHOOL

\begin{tabular}{|c|c|c|}
\hline $\begin{array}{l}\text { No. 68: The Political Economy of Central- } \\
\text { Local Relations in China: Inflation and } \\
\text { Investment Controls During the Reform Era }\end{array}$ & Yasheng Huang & Spring 1997 \\
\hline $\begin{array}{l}\text { No. 67: Between Two Coordination Failures: } \\
\text { Automotive Industrial Policy in China with a } \\
\text { Comparison to Korea }\end{array}$ & Yasheng Huang & Spring 1997 \\
\hline $\begin{array}{l}\text { No. } 66 \text { Published in: Post-Soviet Geography } \\
\text { and Economics, "Red Executives in Russia's } \\
\text { Transition Economy." Vol. 27, No. 10, } \\
\text { November 1996, pp. 633-651. }\end{array}$ & Susan J. Linz & January 1997 \\
\hline $\begin{array}{l}\text { No. } 65 \text { Published in: Industrial and } \\
\text { Corporate Change, "On the Sequencing of } \\
\text { Privatization in Transition Economies." Vol. } \\
7, \text { No. } 1,1998 .\end{array}$ & Gautam Ahuja and Sumit K. Majumdar & April 1997 \\
\hline $\begin{array}{l}\text { No. 64: Published in: Journal of Law and } \\
\text { Economics, "Foreign Ownership and } \\
\text { Profitability: Property Rights, Control and the } \\
\text { Performance of Firms in Indian Industry" } \\
\text { 42(1), April 1999, pp. 209-38. }\end{array}$ & $\begin{array}{l}\text { Pradeep K. Chhibber and Sumit K. } \\
\text { Majumdar }\end{array}$ & April 1997 \\
\hline $\begin{array}{l}\text { No. 63: How Taxing Is Corruption on } \\
\text { International Investors? }\end{array}$ & Shang-Jin Wei & February 1997 \\
\hline $\begin{array}{l}\text { No. 62: What Can We Learn from the } \\
\text { Experience of Transitional Economies with } \\
\text { Labour Market Policies? } \\
\text { No. 61: Published in: Accounting } \\
\text { Organizations and Society, "Economic } \\
\text { Transition, Strategy and the Evolution of } \\
\text { Management Accounting Practices: The Case } \\
\text { of India” 24(5,6), Jul/Aug 1999, pp. 379-412. }\end{array}$ & $\begin{array}{l}\text { Shannon W. Anderson and William N. } \\
\text { Lanen }\end{array}$ & April 1997 \\
\hline $\begin{array}{l}\text { No. 60a: Enterprise Investment During the } \\
\text { Transition: Evidence from Czech Panel Data }\end{array}$ & Lubomír Lizal and Jan Svejnar & December 1997 \\
\hline $\begin{array}{l}\text { No. 59: Published in: Journal of Law, } \\
\text { Economics, and Organization, "Institutional } \\
\text { Environment, Community Government, and } \\
\text { Corporate Governance: Understanding } \\
\text { China's Township-Village Enterprises." } \\
\text { 14(1), April 1998, pages 1-23 }\end{array}$ & Jiahua Che and Yingyi Qian & April 1997 \\
\hline $\begin{array}{l}\text { No. 58: From the Grabbing Hand to the } \\
\text { Helping Hand }\end{array}$ & Jiahua Che & June 2000 \\
\hline $\begin{array}{l}\text { No. 57: Published in: Brookings Papers on } \\
\text { Economic Activity, "The Unofficial Economy } \\
\text { in Transition." 1: 1998. }\end{array}$ & $\begin{array}{l}\text { Simon Johnson, Daniel Kaufmann, and } \\
\text { Andrei Schleifer }\end{array}$ & June 1997 \\
\hline $\begin{array}{l}\text { No. 56: Taxes and Government Incentives: } \\
\text { Eastern Europe vs. China }\end{array}$ & Roger H. Gordon and David D. Li & April 1997 \\
\hline No. 55: Corruption and Reform & Susanto Basu and David Li & June 1996 \\
\hline $\begin{array}{l}\text { No. 54: Decentralization and the } \\
\text { Macroeconomic Consequences of } \\
\text { Commitment to State-Owned Firms }\end{array}$ & Loren Brandt and Xiaodong Zhu & June 1997 \\
\hline $\begin{array}{l}\text { No. 53: Published in: The International } \\
\text { Journal of Industrial Organization, } \\
\text { "Competitive Shocks and Industrial Structure: } \\
\text { The Case of Polish Manufacturing." August, }\end{array}$ & Pankaj Ghemawat and Robert E. Kennedy & May 1997 \\
\hline
\end{tabular}

Publications denoted by an asterisk are not available on the Kresge Library webpage (http://www.wdi.bus.umich.edu) 


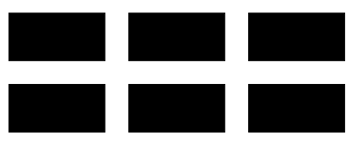

THE WILLIAM DAVIDSON INSTITUTE

AT THE UNIVERSITY OF MICHIGAN BUSINESSSCHOOL

\begin{tabular}{|c|c|c|}
\hline 1999. & & \\
\hline $\begin{array}{l}\text { No. 52: Published in: The Quarterly Journal } \\
\text { of Economics, "Insecure Property Rights and } \\
\text { Government Ownership of Firms." May, } \\
\text { 1998. }\end{array}$ & Jiahua Che and Yingyi Qian & May 1997 \\
\hline $\begin{array}{l}\text { No. 51: Incentives, Scale Economies, and } \\
\text { Organizational Form }\end{array}$ & $\begin{array}{l}\text { Eric Maskin, Yingyi Qian, and Chenggang } \\
X u\end{array}$ & May 1997 \\
\hline $\begin{array}{l}\text { No. 50: Published in: Post-Soviet-Affairs, } \\
\text { "End of the Tunnel? The Effects of Financial } \\
\text { Stabilization in Russia" April-June 1997, } \\
\text { pages 105-33 }\end{array}$ & $\begin{array}{l}\text { Barry W. Ickes, Peter Murrell, and Randi } \\
\text { Ryterman }\end{array}$ & March 1997 \\
\hline $\begin{array}{l}\text { No. 49: The Evolution of Bank Credit Quality } \\
\text { in Transition: Theory and Evidence from } \\
\text { Romania }\end{array}$ & Enrico C. Perotti and Octavian Carare & October 1996 \\
\hline $\begin{array}{l}\text { No. 48: Where Do the Leaders Trade? } \\
\text { Information Revelation and Interactions } \\
\text { Between the Segments of Czech Capital } \\
\text { Markets }\end{array}$ & Jan Hanousek and Libor Nemecek & May 1997 \\
\hline $\begin{array}{l}\text { No. 47: Firms' Heterogeneity in Transition: } \\
\text { Evidence from a Polish Panel Data Set }\end{array}$ & Irena Grosfeld and Jean-François Nivet & May 1997 \\
\hline $\begin{array}{l}\text { No. 46: Strategic Creditor Passivity, } \\
\text { Regulation, and Bank Bailouts }\end{array}$ & Janet Mitchell & May 1997 \\
\hline $\begin{array}{l}\text { No. } 45 \text { a: Decentralization in Transition } \\
\text { Economies: A Tragedy of the Commons? }\end{array}$ & Daniel M. Berkowitz and Wei Li & September 1997 \\
\hline $\begin{array}{l}\text { No. 44a: The Information Content of Stock } \\
\text { Markets: Why do Emerging Markets have } \\
\text { Synchronous Stock Price Movements? } \\
\text { (forthcoming in the Journal of Financial } \\
\text { Economics). } \\
\text { No. 43: Agency in Project Screening and } \\
\text { Termination Decisions: Why Is Good Money } \\
\text { Thrown After Bad? }\end{array}$ & $\begin{array}{l}\text { Randall Morck, Bernard Yeung, and } \\
\text { Wayne Yu }\end{array}$ & $\begin{array}{l}\text { February } 1999 \\
\text { May } 1997\end{array}$ \\
\hline $\begin{array}{l}\text { No. 42: Published in: Economics of } \\
\text { Transition, "Channels of Redistribution: } \\
\text { Inequality and Poverty in the Russian } \\
\text { Transition." Vol. } 7 \text { (2) } 1999 .\end{array}$ & $\begin{array}{l}\text { Simon Commander, Andrei Tolstopiatenko, } \\
\text { and Ruslan Yemtsov }\end{array}$ & May 1997 \\
\hline $\begin{array}{l}\text { No. 41: Published in: Economics of } \\
\text { Transition, "Labour Market Characteristics } \\
\text { and Profitability: Econometric Analysis of } \\
\text { Hungarian Exporting Firms, 1986-1995” } \\
\text { 6(1), May 1998, pages 145-62 }\end{array}$ & László Halpern and Gabor Korosi & May 1997 \\
\hline $\begin{array}{l}\text { No. 40: Published in: the Harvard Law } \\
\text { Review, "The Tragedy of the Anticommons: } \\
\text { Property in the Transition from Marx to } \\
\text { Markets." January 1998. }\end{array}$ & Michael Heller & February 1997 \\
\hline $\begin{array}{l}\text { No. 39: Privatization and Managerial } \\
\text { Efficiency }\end{array}$ & Olivier Debande and Guido Friebel & May 1997 \\
\hline $\begin{array}{l}\text { No. } 38 \text { Published in: The Quarterly Journal } \\
\text { of Economics, “Disorganization.” Vol. 112, } \\
\text { No. 4, November 1997, pp. 1091-1126. }\end{array}$ & Olivier Blanchard and Michael Kremer & January 1997 \\
\hline $\begin{array}{l}\text { No. 37: Published in: Economics of } \\
\text { Transition, "Transition and the Output Fall." }\end{array}$ & Gérard Roland and Thierry Verdier & March 1997 \\
\hline
\end{tabular}

Publications denoted by an asterisk are not available on the Kresge Library webpage (http://www.wdi.bus.umich.edu) 


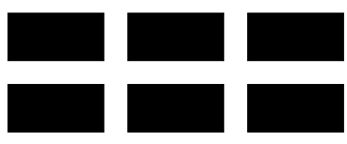

THE WILLIAM DAVIDSON INSTITUTE

AT THE UNIVERSITY OF MICHIGAN BUSINESSSCHOOL

\begin{tabular}{|c|c|c|}
\hline $7(1), 1999$, pages $1-28$. & & \\
\hline $\begin{array}{l}\text { No. 36: Restructuring an Industry During } \\
\text { Transition: A Two-Period Model }\end{array}$ & Richard Ericson & September 1996 \\
\hline $\begin{array}{l}\text { No. 34: The East-West Joint Venture: BC } \\
\text { Torsion Case Study }\end{array}$ & Sonia Ferencikova and Vern Terpstra & December 1998 \\
\hline $\begin{array}{l}\text { No. } 33 \text { Published in: Journal of Comparative } \\
\text { Economics, "Quantifying Price Liberalization } \\
\text { in Russia." Vol. 26, No. 4, December 1998, } \\
\text { pp. } 735-737 .\end{array}$ & $\begin{array}{l}\text { Daniel Berkowitz, David DeJong, and } \\
\text { Steven Husted }\end{array}$ & December 1998 \\
\hline $\begin{array}{l}\text { No. 32: What Can North Korea Learn from } \\
\text { China's Market Reforms? }\end{array}$ & John McMillan & September 1996 \\
\hline $\begin{array}{l}\text { No. 31: Published in: China-Economic- } \\
\text { Review, “Towards a Model of China as a } \\
\text { Partially Reformed Developing Economy } \\
\text { Under a Semifederalist Government.", 9(1), } \\
\text { Spring 1998, pages 1-23. }\end{array}$ & Yijiang Wang and Chun Chang & March 1997 \\
\hline $\begin{array}{l}\text { No. 30: Convergence in Output in Transition } \\
\text { Economies: Central and Eastern Europe, } \\
\text { 1970-1995 }\end{array}$ & Saul Estrin and Giovanni Urga & February 1997 \\
\hline $\begin{array}{l}\text { No. 29: Published in: Economics of } \\
\text { Transition, "Altered Band and Exchange } \\
\text { Volatility." Volume 6, no. 1, 1998, 173-181. }\end{array}$ & Evzen Kocenda & March 1997 \\
\hline $\begin{array}{l}\text { No. 28: Published in: Quarterly Journal of } \\
\text { Economics, "Public Versus Private } \\
\text { Ownership of Firms: Evidence from Rural } \\
\text { China." Volume 113, no. 3, August 1998, 773- } \\
\text { 808. }\end{array}$ & Hehui Jin and Yingyi Qian & January 1997 \\
\hline $\begin{array}{l}\text { No. 27: East-West Joint Ventures in a } \\
\text { Transitional Economy: The Case of Slovakia }\end{array}$ & Sonia Ferencikova & March 1997 \\
\hline $\begin{array}{l}\text { No. 26: Published in Economic Analysis } \\
\text { "Behavior of a Slovenian Firm in Transition" } \\
\text { Vol. 1, no. 1, 1998, 57-73. }\end{array}$ & Janez Prasnikar & February 1997 \\
\hline $\begin{array}{l}\text { No. 25: Cultural Encounters and Claims to } \\
\text { Expertise in Postcommunist Capitalism }\end{array}$ & Michael D. Kennedy & February 1997 \\
\hline $\begin{array}{l}\text { No. 24: ZVU a.s.: Investment Funds on the } \\
\text { Board of Directors of an Engineering Giant }\end{array}$ & Tory Wolff & August 1995 \\
\hline $\begin{array}{l}\text { No. 23: The Role of Investment Funds in the } \\
\text { Czech Republic (joint publication with Czech } \\
\text { Management Center) }\end{array}$ & Dusan Triska & June 1996 \\
\hline $\begin{array}{l}\text { No. 22: Czech Investment Fund Industry: } \\
\text { Development and Behaviour (joint publication } \\
\text { with Czech Management Center) }\end{array}$ & Richard Podpiera & May 1996 \\
\hline $\begin{array}{l}\text { No. 21: Restructuring of Czech Firms: An } \\
\text { Example of Gama, a.s. (joint publication with } \\
\text { Czech Management Center) }\end{array}$ & Antonin Bulin & June 1996 \\
\hline $\begin{array}{l}\text { No. 20: YSE Funds: A Story of Czech } \\
\text { Investment Funds (joint publication with } \\
\text { Czech Management Center) }\end{array}$ & Michal Otradovec & November 1995 \\
\hline $\begin{array}{l}\text { No. 19: První Investicni a.s., The First } \\
\text { Investment Corporation (joint publication } \\
\text { with Czech Management Center) }\end{array}$ & Jaroslav Jirasek & August 1995 \\
\hline No. 18: PPF a.s., The First Private Investment & Michal Otradovec & November 1995 \\
\hline
\end{tabular}

Publications denoted by an asterisk are not available on the Kresge Library webpage (http://www.wdi.bus.umich.edu) 


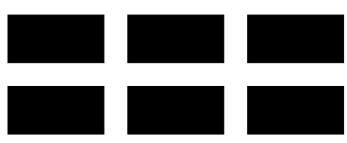

THE WILLIAM DAVIDSON INSTITUTE

AT THE UNIVERSITY OF MICHIGAN BUSINESSSCHOOL

\begin{tabular}{|c|c|c|}
\hline $\begin{array}{l}\text { Fund (joint publication with Czech } \\
\text { Management Center) }\end{array}$ & & \\
\hline $\begin{array}{l}\text { No. } 17 \text { Published in: Post-Soviet Geography } \\
\text { and Economics, “Russia's Managers in } \\
\text { Transition: Pilferers or Paladins?" Vol. } 37 \text {, } \\
\text { o.7 (September 1996), pp. } 397-426 .\end{array}$ & Susan J. Linz and Gary Krueger & November 1996 \\
\hline $\begin{array}{l}\text { No. 16: Banks in Transition-Investment } \\
\text { Opportunities in Central Europe and Russia } \\
\text { Edited Transcript from } 31 \text { May } 1996 \\
\text { Conference in New York City }\end{array}$ & $\begin{array}{l}\text { With commentary and edited by Anna } \\
\text { Meyendorff }\end{array}$ & January 1997 \\
\hline $\begin{array}{l}\text { No. 15: Marketing in Transitional Economies: } \\
\text { Edited Transcript \& Papers from } 1 \text { April } 1996 \\
\text { Conference in Ann Arbor, Michigan }\end{array}$ & Compiled by The Davidson Institute & December 1996 \\
\hline $\begin{array}{l}\text { No. 14: Pensions in the Former Soviet Bloc: } \\
\text { Problems and Solutions. Published by } \\
\text { Council on Foreign Relations. "The Coming } \\
\text { Global Pension Crisis" New York, } 1997\end{array}$ & Jan Svejnar & November 1996 \\
\hline $\begin{array}{l}\text { No. 13: Enterprise Restructuring and } \\
\text { Performance in the Transition. Forthcoming } \\
\text { in Financial Systems in Transition: The } \\
\text { Design of Financial Systems in Central } \\
\text { Europe eds. Anna Meyendorff and Anjan } \\
\text { Thakor. }\end{array}$ & $\begin{array}{l}\text { Lubomir Lizal, Miroslav Singer, and Jan } \\
\text { Svejnar }\end{array}$ & December 1996 \\
\hline $\begin{array}{l}\text { No. } 12 \text { Published in: Journal of International } \\
\text { Marketing, "Executive Insights: Marketing } \\
\text { Issues and Challenges in Transitional } \\
\text { Economies." Vol. 5, No. 4, 1997, pp. 95-114. } \\
\text { Also published in: Marketing Issues in } \\
\text { Transitional Economies ed. Rajeev Batra. }\end{array}$ & Rajeev Batra & April 1997 \\
\hline $\begin{array}{l}\text { No. 11: Worker Trust and System } \\
\text { Vulnerability in the Transition from Socialism } \\
\text { to Capitalism }\end{array}$ & Andrew Schotter & August 1996 \\
\hline $\begin{array}{l}\text { No. } 10 \text { Published in: Comparative Economic } \\
\text { Studies, "Russian Firms in Transition: } \\
\text { Champions, Challengers, and Chaff." Vol. } \\
\text { 39, No.2, Summer 1997, pp. 1-36. }\end{array}$ & Susan J. Linz & July 1996 \\
\hline $\begin{array}{l}\text { No. 9: Corporate Debt Crisis and Bankruptcy } \\
\text { Law During the Transition: The Case of China }\end{array}$ & David D. Li and Shan Li & December 1995 \\
\hline $\begin{array}{l}\text { No. } 8 \text { Published in: Journal of Comparative } \\
\text { Economics, “A Theory of Ambiguous } \\
\text { Property Rights in Transition Economies: The } \\
\text { Case of the Chinese Non-State Sector.” Vol. } \\
\text { 23, No. 1, August 1996, pp. 1-19. }\end{array}$ & David D. Li & June 1996 \\
\hline $\begin{array}{l}\text { No. 7: The Foreign Economic Contract Law of } \\
\text { China: Cases and Analysis }\end{array}$ & Dong-lai Li & June 1993 \\
\hline $\begin{array}{l}\text { No. 3: Bank Privatization in Hungary and the } \\
\text { Magyar Kulkereskedelmi Bank Transaction }\end{array}$ & Roger Kormendi and Karen Schnatterly & May 1996 \\
\hline $\begin{array}{l}\text { Replacing Nos. 1-2 \& 4-6: Journal of } \\
\text { Comparative Economics Symposium on } \\
\text { "Bank Privatization in Central Europe and } \\
\text { Russia." Vol. 25, No. 1, August 1997. }\end{array}$ & $\begin{array}{l}\text { No. } 1 \text { "Bank Privatization in Transitional } \\
\text { Economies" by Roger Kormendi and } \\
\text { Edward Snyder. No. } 2 \text { "Transactional } \\
\text { Structures of Bank Privatizations in } \\
\text { Central Europe and Russia” by Anna }\end{array}$ & August 1997 \\
\hline
\end{tabular}

Publications denoted by an asterisk are not available on the Kresge Library webpage (http://www.wdi.bus.umich.edu) 


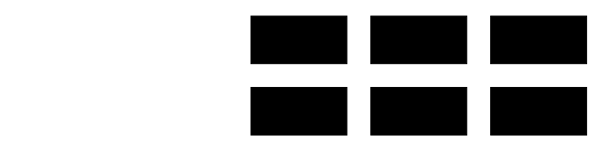

THE WILLIAM DAVIDSON INSTITUTE

AT THE UNIVERSITY OF MICHIGAN BUSINESSSCHOOL

\begin{tabular}{|l|l|}
\hline & Meyendorff and Edward A. Snyder. No. 4 \\
& "Bank Privatization in Poland: The Case \\
of Bank Slaski" by Jeffery Abarbaness and & \\
& John Bonin. No. 5 "Bank Privatization in \\
& Post-Communist Russia: The Case of \\
& Zhilsotsbank" by Jeffery Abarbanell and \\
& Anna Meyendorff and No.6 “"The Czech \\
& Republic's Commercial Bank: Komercni \\
& Banka" by Edward A. Snyder and Roger \\
& C. Kormendi. \\
\hline
\end{tabular}

Publications denoted by an asterisk are not available on the Kresge Library webpage (http://www.wdi.bus.umich.edu) 